\title{
Species composition of climbers in seasonal semideciduous forest fragments of Southeastern Brazil
}

\author{
Karin dos Santos ${ }^{1,4}$, Luiza Sumiko Kinoshita ${ }^{2}$ \& Andréia Alves Rezende ${ }^{3}$ \\ ${ }^{1}$ Department of Phanerogamic Botany, \\ Swedish Museum of Natural History, SE-104 05, Stockholm, Sweden \\ ${ }^{2}$ Departamento de Botânica, Instituto de Biologia, \\ Universidade Estadual de Campinas - UNICAMP, \\ CP 6109, CEP 13083-970, Campinas, SP, Brazil \\ ${ }^{3}$ Departamento de Ciências Biológicas, Centro Universitário de Rio Preto, \\ Rua Yvette Gabriel Atique, 45, CEP 15025-400, São José do Rio Preto, SP, Brazil \\ ${ }^{4}$ Author for correspondence: Karindos Santos, e-mail: karin.santos@nrm.se
}

SANTOS, K., KINOSHITA, L. S. \& REZENDE, A. A. Species composition of climbers in seasonal semideciduous forest fragments of Southeastern Brazil. Biota Neotrop., 9 (4): http://www.biotaneotropica. org.br/v9n4/en/abstract?inventory+bn00309042009.

\begin{abstract}
In this study we evaluated floristic composition patterns of communities of climbers within ten inventories carried out in semideciduous forest fragments of southeastern Brazil. One of the inventories is original, being carried out for the present study in Ribeirão Cachoeira forest, Campinas, São Paulo State, Southeastern Brazil. This inventory was then pooled together to other nine climbers' inventories made in other forests of Southeastern Brazil to form a data base, which was examined regarding species richness, similarity, species distribution and climbing methods. The total number of species obtained was 355, belonging to 145 genera and 43 families. The ten most diverse families Bignoniaceae (45 species), Fabaceae (42), Malpighiaceae (36), Asteraceae (31), Apocynaceae (29), Sapindaceae (28), Convolvulaceae (21), Cucurbitaceae (14), Passifloraceae (10), and Euphorbiaceae (8) contributed to $74.4 \%$ of the total number of species recorded. The commonest climbing method in the studied sites was main stem or branch twining, accounting for 178 species or $50.1 \%$ of the total, the second commonest was tendril climbing (121 species, $34.1 \%$ ), and the least, scrambling (56 species, 15.8\%). We found a high percentage of exclusive species i.e., those occurring in only one forest site, which accounted for $49.3 \%$ of the total recorded. The mean similarity among forest sites (30\%) may be considered low. The climbing species contribution to the total wood plant richness recorded on the forests sites was very high in some of the sites (up to 52.5\%). These results indicated the importance of climber communities to plant diversity for semideciduous forests in Southeastern Brazil, enhancing the regional diversity and the conservation value of these forest remnants.
\end{abstract}

Keywords: lianas, climbing mechanism, floristic, similarity, NMS.

SANTOS, K., KINOSHITA, L. S. \& REZENDE, A. A. Composição florística de trepadeiras em florestas sazonais semidecíduais do sudeste do Brasil. Biota Neotrop., 9 (4): http://www.biotaneotropica.org.br/v9n4/ en/abstract?inventory+bn00309042009.

Resumo: No presente estudo avaliamos as variações na composição de espécies das comunidades de trepadeiras abordando dez inventários florísticos realizados em fragmentos de floresta estacional semidecidual do sudeste do Brasil. Um destes inventários é inédito e foi realizado especialmente para o presente estudo. Comparamos este levantamento a outros nove inventários florísticos de trepadeiras realizados em outras florestas do sudeste para investigar aspectos como riqueza, similaridade, distribuição de espécies e mecanismos de escalada. Ao todo foram encontradas 355 espécies, pertencentes a 145 gêneros e 43 famílias. As dez famílias mais ricas Bignoniaceae (45 espécies), Fabaceae (42), Malpighiaceae (36), Asteraceae (31), Apocynaceae (29), Sapindaceae (28), Convolvulaceae (21), Cucurbitaceae (14), Passifloraceae (10), e Euphorbiaceae (8) representaram 74,4\% do total de espécies encontrado. Considerando os mecanismos de escalada, observamos o predomínio de espécies volúveis (178 espécies ou 50,1\%), sobre espécies com gavinhas (121 espécies, 34,1\%), e apoiadoras (56 espécies, 15,8\%). A porcentagem de espécies exclusivas, i.e. aquelas que ocorreram em apenas uma das localidades estudadas, foi de 49,3\% do total amostrado e pode ser considerada alta. Além disso, a similaridade entre as florestas analisadas foi bastante baixa e a contribuição das espécies de trepadeiras para a diversidade de plantas lenhosas nestas áreas foi bastante elevada em algumas áreas, chegando a 52,5\%. Estes resultados indicaram a importância das comunidades de trepadeiras para a diversidade vegetal nas florestas semideciduais do sudeste do Brasil, aumentando a valor de conservação desses remanescentes florestais.

Palavras-chave: lianas, mecanismos de escalada, ordenação NMS, similaridade. 


\section{Introduction}

The semideciduous seasonal forest of the Southeastern Brazil is probably one of the most threatened and fragmented ecosystems on earth. The maintenance of its biodiversity depends on the conservation of small fragments spread over an agricultural landscape. The biological studies on these forest remnants have improved a lot during the last three decades, enhancing the required background for management and conservation actions. However, climbers' communities have been neglected by most of those studies, which focused mainly on trees. This study comes as a small contribution towards fulfilling this gap.

Climbers are an abundant and diverse group of plants in forests throughout the world, particularly in the tropics. They can constitute $\sim 25 \%$ of woody stem density and represent from 25 to $44 \%$ of the woody species diversity in tropical forests (Gentry 1991, Pérez-Salicrup et al. 2001), providing a remarkable food source for the associated fauna and, by physically linking trees together, furnishing canopy-to-canopy access for arboreal animals (Emmons \& Gentry 1983, Morellato \& Leitão Filho 1996). Thus, the importance of improving the knowledge about lianas (woody climbers) lies on the crucial ecological role they play in many aspects of tropical forest dynamics.

Lianas are commonly seen as structural parasites, invaders or weedy species, which respond positively to increased $\mathrm{CO} 2$ concentrations, light penetration and forest disturbance, and consequently thrive in fragmented/disturbed landscapes (Putz 1984, Laurance 1991, 1997, Laurance et al. 2001, Phillips et al. 2002). When abundant, this life form is considered to be an inconvenient for economic and conservational reasons. Abundant lianas can reduce the sylvicultural value of a forest by reducing tree growth and fecundity, and increasing tree mortality and stem deformation (Stevens 1987, Putz 1991, Pérez-Salicrup \& Barker 2000, Phillips et al. 2005). Additionally, tree supporting lianas are more prone to break and cause logging damage on their neighbours due to intercrown connections between adjacent trees, enhancing tree turnover in heavy infested forests (Appanah \& Putz 1984, Vidal et al. 1997, Phillips et al. 2005). Lianas can also arrest or suppress gap-phase regeneration, and harm some species while promoting others (Schnitzer et al. 2000). All these factors indicate that forest dynamics and composition can be altered due to lianas' influence, with implications for forest's conservation and sustainability (Putz 1984, Stevens 1987, Pérez-Salicrup \& Barker 2000, Schnitzer et al. 2000, Schnitzer \& Carson 2001, Phillips et al. 2002, 2005).

Few quantitative and qualitative data are available to evaluate these claims in the semideciduous seasonal forests of southeasthern Brazil. This ecosystem is facing an extremely high anthropogenic pressure given its high degree of forest fragmentation, disturbance history, and location, among the larger cities and the most industrialized region of Brazil. Heavy liana infestations are expected for this region, however, little is known about variations in their communities and the contribution of their species to overall diversity in these forests (but see Udulutsch et al. 2004, Tibiriçá et al. 2006, Rezende et al. 2007). Further investigations and data are highly desirable and required to address these questions in semideciduous seasonal forests of southeasthern Brazil (Udulutsch et al. 2004). Floristic inventories are the first step towards a better understanding of climbers' communities in this region; they provide the basic information for further investigations and to support management and conservation policies.

The goal of this study is to contribute to increase the knowledge of communities of climbers in the Southeastern Brazil by describing their floristic composition in a semideciduous seasonal forest fragment of Campinas municipality, State of São Paulo, and providing a preliminary evaluation of the floristic patterns of their communities by comparing the current floristic inventory to other nine inventories carried out in different semideciduous forest fragments of Southeastern Brazil.

\section{Material and Methods}

The tropical semidecidous forest from Southeastern Brazil occurs in areas with rainfall between 1,250 and $2,550 \mathrm{~mm}$. The vegetation is characterized by dense forests with high tree species diversity. The average canopy stature is $15 \mathrm{~m}$ with emergent trees reaching up to $35 \mathrm{~m}$ tall. There are two distinct seasons, the rainy summer, from December through February and the dry winter, from June through August. During the dry season approximately $40 \%$ of the trees composing the canopy are deciduous to some degree (Morellato \& Leitão Filho 1992). The canopy is commonly dominated by families Myrtaceae, Fabaceae, Meliaceae, Apocynaceae and Euphorbiaceae. The most dominant species are Esenbeckia leiocarpa Engl.,
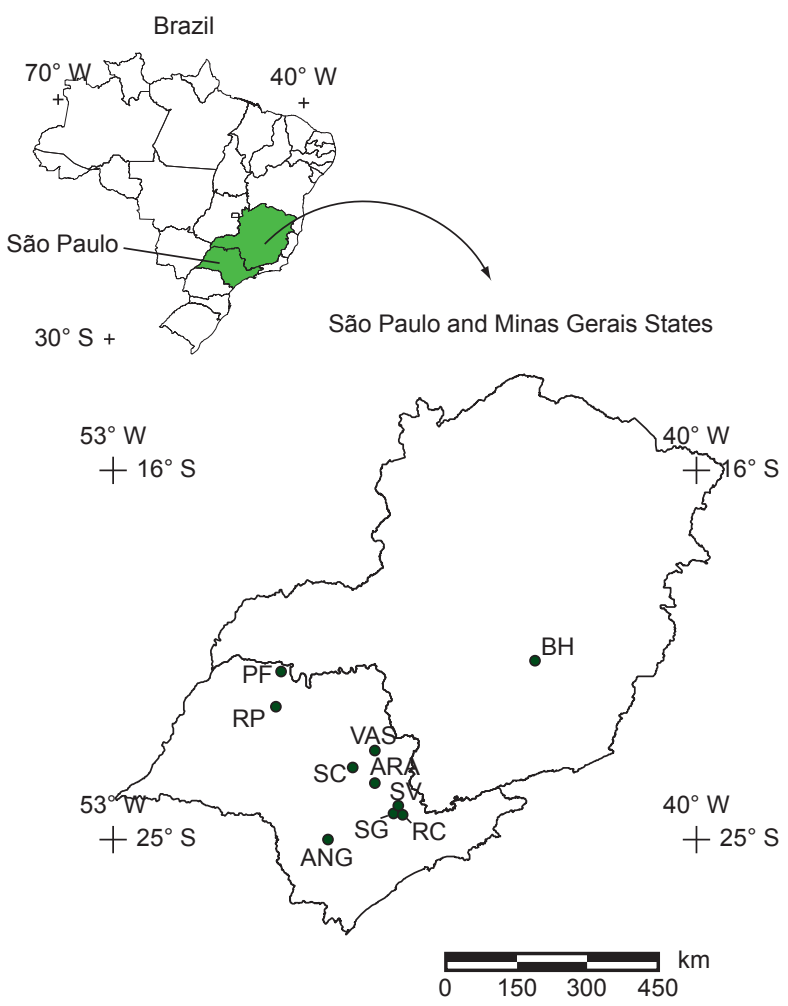

Figure1. Locations of the 10 semideciduous forest sites of southeastern Brazil. ANG = Angatuba (Torres 1989); ARA = Araras (Udulutsch et al. 2004); BH = Belo Horizonte (Lombardi et al. 1999); RC = Ribeirão Cachoeira forest (Present study); RP = São José do Rio Preto (Rezende \& Ranga 2005); PF = Paulo de Faria (Rezende et al. 2007); SC = São Carlos (Hora \& Soares 2002); SG = Santa Genebra (Morellato \& Leitão Filho 1996); SV = Fazenda São Vicente (Bernacci \& Leitão Filho 1996); VAS = Vassununga (Tibiriçá et al. 2006).

Figura 1. Localização dos dez fragmentos de floresta estacional semidecidual estudados no sudeste do Brasil. ANG = Angatuba (Torres 1989); ARA = Araras (Udulutsch et al. 2004); BH = Belo Horizonte (Lombardi et al. 1999); RC = Mata Ribeirão Cachoeira (Presente estudo); RP = São José do Rio Preto (Rezende \& Ranga 2005); PF = Paulo de Faria (Rezende et al. 2007); SC = São Carlos (Hora \& Soares 2002); SG = Santa Genebra (Morellato \& Leitão Filho 1996); SV = Fazenda São Vicente (Bernacci \& Leitão Filho 1996); VAS = Vassununga (Tibiriçá et al. 2006). 
Piptadenia gonoacantha (Mart.) J.F.Macbr., Trichilia clausseni C.DC, Croton floribundus Spreng., Gallesia integrifolia (Spreng.) Harms, Lonchocarpus muehlbergianus Hassl., and Aspidosperma polyneuron Müll.Arg. (K. Santos et al. unpubl. data).

The floristic inventory of the present study was carried out at the Ribeirão Cachoeira Forest, which is the second largest (244.9 ha) of the former continuous semideciduous seasonal forest of Campinas municipality, State of São Paulo. The species composition of climbers was sampled randomly, by walking along the network of paths within the forest fragment and also at forest edges. Every climber found in reproductive state was collected for later identification and recorded for the checklist. Collecting was done mainly from March through December 1996. Identification followed the usual taxonomic techniques and vouchers have been deposited in the UEC Herbarium (Department of Botany, Institute of Biology, State University of Campinas - UNICAMP). Family classification followed APG II (APG 2003).

There are many different kinds of climbers, differing in climbing strategy, morphology and ecology (Gentry 1991). In this study we included woody, subwoody and herbaceous climbing plants which can be defined as plants that begin their life cycles as seedlings rooted in the ground and rely on other plants for physical support in order to reach the top of the forest canopy, never loosing the contact with the ground as they keep rooted in the soil permanently. This means that we excluded hemiepiphytes and epiphytes. Nonetheless, because the majority of the species being considered in this study is woody and also because the other inventories treated here followed the same definition, we may also refer to the studied group of plants using the more widespread term lianas, especially when referring to available literature focusing the theme.

The obtained list of species plus other nine floristic inventories of climbers carried out in different locations of the southeastern Brazil were pulled together to build a presence/absence main matrix data.
Only forests belonging to the seasonal semideciduous forests domain were included, being eight forest inventories from the interior of the São Paulo state and one from the State of Minas Gerais (Figure 1, Table 1). The sampling methods and criteria of inclusion adopted by each study included in the main matrix data varied little, most of them adopted the same methods as in the current inventory (Table 1).

Only taxa identified up to species level were included in the presence/absence main matrix, and the full list was checked for synonymies among taxa. For all species recorded on the main matrix, the climbing mechanisms were determined based on field observation, personal knowledge and/or reference to literature. Species were classified into one of the following categories according to Putz (1984) and Hegarty (1991): a) tendril climbers, b) main stem or branch twiners, and d) scramblers.

We examined the patterns of climbers composition using nonmetric multidimensional scaling (NMS) ordination in PC-ORD version 4.0 (McCune \& Mefford 1999). The ordinations were based on the Sorenson's similarity matrices of species presence-absence data. We also accessed liana species' contribution to the total wood plant richness recorded on the studied forests sites by calculating the proportion of climber species in relation to the total wood species number (trees, shrubs and climbers). The number of trees/shrubs species in each site was also compiled from the literature for most studied sites, with exception of Belo Horizonte (BH), São José do Rio Preto (RP), and Vassununga (VAS).

\section{Results}

Considering all the forests inventories pulled together, we found a total of 355 species of climbers belonging to 145 genera and 43 families (Table 2). The ten most diverse families were Bignoniaceae (45 species), Fabaceae (42), Malpighiaceae (36), Asteraceae (31), Apocynaceae (29), Sapindaceae (28), Convolvulaceae (21),

Table 1. List of the ten semideciduous seasonal forests where liana inventories were conducted in southeastern Brazil.

Tabela 1. Lista dos dez inventários florísticos de lianas realizados em florestas estacionais semideciduais do sudeste do Brasil.

\begin{tabular}{|c|c|c|c|c|c|}
\hline Forest site & Coordinates & Area $^{a}$ & Sample method & 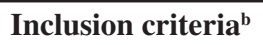 & Reference \\
\hline Angatuba (ANG) & $23^{\circ} 29^{\prime} \mathrm{S}$ and $48^{\circ} 45^{\prime} \mathrm{W}$ & 1394 & Random walks & $\begin{array}{l}\text { Reproductive } \\
\text { material }\end{array}$ & Torres (1989) \\
\hline Araras (ARA) & $22^{\circ} 21^{\prime} \mathrm{S}$ and $47^{\circ} 28^{\prime} \mathrm{W}$ & 230 & Random walks & $\begin{array}{l}\text { Reproductive } \\
\text { material }\end{array}$ & Udulutsch et al. (2004) \\
\hline Belo Horizonte $(\mathrm{BH})$ & $19^{\circ} 52^{\prime} \mathrm{S}$ and $43^{\circ} 58^{\prime} \mathrm{W}$ & 150 & Plots & $\mathrm{DBH} \geq 0.5 \mathrm{~cm}$ & Lombardi et al. (1999) \\
\hline Paulo de Faria (PF) & $19^{\circ} 55^{\prime} \mathrm{S}$ and $49^{\circ} 32^{\prime} \mathrm{W}$ & 650 & Plots & $\mathrm{DBH} \geq 1 \mathrm{~cm}$ & $\begin{array}{l}\text { Rezende et al. (2007); } \\
\text { Stranghetti \& Ranga (1998) }\end{array}$ \\
\hline Ribeirão Cachoeira (RC) & $22^{\circ} 49^{\prime} \mathrm{S}$ and $46^{\circ} 55^{\prime} \mathrm{W}$ & 244.9 & Random walks & $\begin{array}{l}\text { Reproductive } \\
\text { material }\end{array}$ & Present study \\
\hline Santa Genebra (SG) & $22^{\circ} 44^{\prime} \mathrm{S}$ and $47^{\circ} 06^{\prime} \mathrm{W}$ & 250 & Random walks & $\begin{array}{l}\text { Reproductive } \\
\text { material }\end{array}$ & $\begin{array}{l}\text { Morellato \& Leitão Filho } \\
1996\end{array}$ \\
\hline São Carlos (SC) & $21^{\circ} 57^{\prime} \mathrm{S}$ and $47^{\circ} 50^{\prime} \mathrm{W}$ & 112 & Plots & $\mathrm{DBH} \geq 2.5 \mathrm{~cm}$ & Hora \& Soares 2002 \\
\hline São José do Rio Preto (RP) & $20^{\circ} 48^{\prime} \mathrm{S}$ and $49^{\circ} 22^{\prime} \mathrm{W}$ & 168.63 & Random walks & $\begin{array}{l}\text { Reproductive } \\
\text { material }\end{array}$ & Rezende \& Ranga 2005 \\
\hline São Vicente (SV) & $22^{\circ} 55^{\prime} \mathrm{S}$ and $47^{\circ} 03^{\prime} \mathrm{W}$ & 70 & $\begin{array}{l}\text { Random walks/ } \\
\text { Plots }\end{array}$ & Unspecified & $\begin{array}{l}\text { Bernacci \& Leitão Filho } \\
1996\end{array}$ \\
\hline Vassununga (VAS) & $21^{\circ} 41^{\prime} \mathrm{S}$ and $47^{\circ} 34^{\prime} \mathrm{W}$ & 127.08 & Random walks & $\begin{array}{l}\text { Reproductive } \\
\text { material }\end{array}$ & Tibiriçá et al. 2006 \\
\hline
\end{tabular}

${ }^{a}=$ Forest fragment size in hectares; ${ }^{b}=$ this column refers to the criteria adopted by each study to select the plants to be included in the survey. Where $\mathrm{DBH}=$ diameter at breast high; Reproductive material = all the plant stems were collected as long as they were found in reproductive state (flowering or fruiting).

${ }^{\mathrm{a}}=$ Área do fragmento florestal em hectares; ${ }^{\mathrm{b}}=$ esta coluna se refere ao critério de inclusão adotado na amostragem. Onde DBH = diâmetro à altura do peito; Reproductive material = todas as plantas encontradas em estado reprodutivo (flor ou fruto) foram coletadas. 
Table 2. List of 43 families and 355 liana species occurring at the 10 semideciduous forest sites of Southeastern Brazil (See Table 1 and Figure 1 for sites legend). *Climbing methods, where ten $=$ tendril climbers; twi $=$ branch/stem twiners; $s c r=$ scramblers. $\mathrm{x}=$ indicates species occurrence.

Tabela 2. Lista das 43 famílias e 355 espécies de lianas encontradas nos dez fragmentos de floresta estacional semidecidual estudados no sudeste do Brasil (Siglas dos fragmentos como na Figura 1 e Tabela 1). *Mecanismos de escalada, onde ten= gavinhas; twi = volúveis; scr = escandentes. X = ocorrência da espécie.

\begin{tabular}{|c|c|c|c|c|c|c|c|c|c|c|c|}
\hline Family and species & Climb* & ANG & ARA & BH & PF & $\mathbf{R C}$ & $\mathbf{R P}$ & SC & SG & SV & VAS \\
\hline \multicolumn{12}{|l|}{ ACANTHACEAE } \\
\hline Mendoncia puberula Mart. & twi & - & $\mathrm{x}$ & - & - & - & - & - & $\mathrm{x}$ & - & - \\
\hline Mendoncia velloziana Mart. & twi & - & $\mathrm{x}$ & - & - & - & - & - & $\mathrm{x}$ & - & - \\
\hline \multicolumn{12}{|l|}{ AGAVACEAE } \\
\hline Herreria salsaparilha Mart. & twi & - & - & - & $\mathrm{x}$ & $\mathrm{x}$ & $\mathrm{x}$ & - & - & - & - \\
\hline \multicolumn{12}{|l|}{ ALSTROEMERIACEAE } \\
\hline Alstroemeria nemorosa Gardner & twi & - & $\mathrm{x}$ & - & - & - & - & - & - & - & - \\
\hline Bomarea martiana Schen. & $\mathrm{scr}$ & $\mathrm{x}$ & - & - & - & - & - & - & - & - & - \\
\hline \multicolumn{12}{|l|}{ AMARANTHACEAEE } \\
\hline Chamissoa altissima (Jacq.) Kunth & scr & $\mathrm{x}$ & $\mathrm{x}$ & - & $\mathrm{x}$ & - & - & - & $\mathrm{x}$ & $\mathrm{x}$ & $\mathrm{x}$ \\
\hline Hebanthe paniculata Mart. & twi & $\mathrm{x}$ & - & - & $\mathrm{x}$ & $\mathrm{x}$ & $\mathrm{x}$ & - & $\mathrm{x}$ & $\mathrm{x}$ & $\mathrm{x}$ \\
\hline \multicolumn{12}{|l|}{ APOCYNACEAE } \\
\hline Araujia sericifera Brot. & twi & - & - & - & - & - & $\mathrm{x}$ & - & - & - & - \\
\hline Calotropis procera (Aiton) R.Br. & $\mathrm{scr}$ & - & - & - & - & - & - & - & - & - & $\mathrm{x}$ \\
\hline Condylocarpon isthmicum (Vell.) A.DC. & twi & $\mathrm{x}$ & $\mathrm{x}$ & - & - & $\mathrm{x}$ & - & $\mathrm{x}$ & $\mathrm{x}$ & - & $\mathrm{x}$ \\
\hline Ditassa anomala Mart. & twi & - & - & - & - & - & - & - & $\mathrm{x}$ & - & - \\
\hline Forsteronia glabrescens Müll.Arg. & twi & - & - & - & - & - & $\mathrm{x}$ & - & - & - & - \\
\hline Forsteronia leptocarpa (Hook. \& Arn.) A.DC. & twi & - & $\mathrm{x}$ & - & - & - & - & - & $\mathrm{x}$ & - & - \\
\hline Forsteronia pilosa (Vell.) Müll.Arg. & twi & - & $\mathrm{x}$ & - & $\mathrm{x}$ & $\mathrm{x}$ & - & - & - & - & $\mathrm{x}$ \\
\hline Forsteronia pubescens A.DC. & twi & - & $\mathrm{x}$ & - & $\mathrm{x}$ & $\mathrm{x}$ & $\mathrm{x}$ & $\mathrm{x}$ & - & - & $\mathrm{x}$ \\
\hline Forsteronia refracta Müll.Arg. & twi & - & $\mathrm{x}$ & - & - & - & - & - & - & - & $\mathrm{x}$ \\
\hline Forsteronia rufa Müll.Arg. & twi & - & - & - & - & - & - & - & $\mathrm{x}$ & - & - \\
\hline Forsteronia velloziana (A.DC.) Woodson & twi & - & - & $\mathrm{x}$ & - & - & - & - & - & - & - \\
\hline Gonioanthela hilariana (E.Fourn.) Malme & twi & - & $\mathrm{x}$ & - & - & - & - & - & - & - & - \\
\hline Gonolobus rostratus (Vahl) Schult. & twi & - & - & - & - & - & - & - & $\mathrm{x}$ & - & - \\
\hline Gonolobus selloanus (E.Fourn.) Bacigalupo & twi & - & $\mathrm{x}$ & - & - & - & - & - & - & - & - \\
\hline Mesechites mansoanus (A.DC.) Woodson & twi & - & - & - & - & - & $\mathrm{x}$ & - & - & - & - \\
\hline Orthosia urceolata E.Fourn. & twi & - & $\mathrm{x}$ & - & - & - & - & - & - & - & - \\
\hline Oxypetalum appendiculatum Mart. & twi & - & - & - & - & - & - & - & $\mathrm{x}$ & - & - \\
\hline Oxypetalum balansae Malme & twi & - & - & - & - & - & $\mathrm{x}$ & - & - & - & - \\
\hline Oxypetalum erianthum Decne. & twi & - & - & - & $\mathrm{x}$ & - & $\mathrm{x}$ & - & - & - & - \\
\hline Oxypetalum molle Hook. \& Arn. & twi & - & - & - & - & - & - & - & $\mathrm{x}$ & - & - \\
\hline Prestonia coalita (Vell.) Woodson & twi & - & $\mathrm{x}$ & - & $\mathrm{x}$ & $\mathrm{x}$ & $\mathrm{x}$ & - & $\mathrm{x}$ & $\mathrm{x}$ & $\mathrm{x}$ \\
\hline Prestonia dusenii (Malme) Woodson & twi & - & - & - & - & - & $\mathrm{x}$ & - & - & - & - \\
\hline Prestonia lagoensis (Müll.Arg.) Woodson & twi & - & - & - & $\mathrm{x}$ & - & - & - & - & - & - \\
\hline Prestonia riedelii (Müll.Arg.) Markgr. & twi & - & - & - & - & - & - & - & $\mathrm{x}$ & - & - \\
\hline Prestonia tomentosa $\mathrm{R} . \mathrm{Br}$. & twi & - & - & - & $\mathrm{x}$ & $\mathrm{x}$ & $\mathrm{x}$ & - & $\mathrm{x}$ & - & - \\
\hline Sarcostemma clausum (Jacq.) Schult. & twi & - & - & - & - & - & - & - & - & - & $\mathrm{x}$ \\
\hline Schubertia grandiflora Mart. \& Zucc. & twi & - & - & - & - & $\mathrm{x}$ & $\mathrm{x}$ & - & - & - & - \\
\hline Secondatia densiflora A.DC. & twi & - & - & - & $\mathrm{x}$ & - & $\mathrm{x}$ & - & - & - & - \\
\hline Tassadia propinqua Decne. & twi & - & - & - & - & - & - & - & $\mathrm{x}$ & - & - \\
\hline \multicolumn{12}{|l|}{ ARACEAE } \\
\hline Philodendron propinquum Schott & twi & - & - & - & - & - & - & - & - & $\mathrm{x}$ & - \\
\hline \multicolumn{12}{|l|}{ ARISTOLOCHIACEAE } \\
\hline Aristolochia arcuata Mast. & twi & - & $\mathrm{x}$ & - & - & $\mathrm{x}$ & - & - & $\mathrm{x}$ & - & - \\
\hline Aristolochia esperanzae Kuntze & twi & - & - & - & - & - & $\mathrm{x}$ & - & - & - & - \\
\hline Aristolochia galeata Mart. & twi & $\mathrm{x}$ & $\mathrm{x}$ & - & - & - & - & - & $\mathrm{x}$ & - & - \\
\hline Aristolochia melastoma Silva Manso ex Duch. & twi & - & $\mathrm{x}$ & - & - & - & - & - & $\mathrm{x}$ & $\mathrm{x}$ & - \\
\hline
\end{tabular}


Table 2. Continued...

\begin{tabular}{|c|c|c|c|c|c|c|c|c|c|c|c|}
\hline Family and species & Climb* & ANG & ARA & BH & PF & $\mathbf{R C}$ & $\mathbf{R P}$ & $\mathrm{SC}$ & SG & SV & VAS \\
\hline \multicolumn{12}{|l|}{ ASTERACEAE } \\
\hline Baccharis quitensis Kunth & scr & - & $\mathrm{x}$ & - & - & - & - & - & - & - & $\mathrm{x}$ \\
\hline Bidens brasiliensis Sherff & twi & - & - & - & - & - & - & - & $\mathrm{x}$ & - & - \\
\hline Bidens squarrosa Kunth & twi & - & $\mathrm{x}$ & - & - & - & - & - & - & - & - \\
\hline Calea pinnatifida (R.Br.) Less. & scr & $\mathrm{x}$ & $\mathrm{x}$ & - & - & $\mathrm{x}$ & - & - & $\mathrm{x}$ & - & - \\
\hline $\begin{array}{l}\text { Chromolaena maximilianii } \\
\text { (Schrader) R.M.King \& H.Rob. }\end{array}$ & scr & - & $\mathrm{x}$ & - & - & - & - & - & - & - & - \\
\hline Cyrtocymura scorpioides (Lam.) H.Rob. & scr & - & $\mathrm{x}$ & - & - & $\mathrm{x}$ & - & - & - & - & $\mathrm{x}$ \\
\hline Dasyphyllum brasiliense (Spreng.) Cabrera & scr & $\mathrm{x}$ & - & - & $\mathrm{x}$ & $\mathrm{x}$ & $\mathrm{x}$ & - & $\mathrm{x}$ & - & - \\
\hline Dasyphyllum flagellare (Casar.) Cabrera & scr & $\mathrm{x}$ & - & - & - & - & - & - & - & - & - \\
\hline Dasyphyllum spinescens (Less.) Cabrera & scr & - & - & - & - & - & - & - & $\mathrm{x}$ & $\mathrm{x}$ & - \\
\hline Dasyphyllum synacanthum (Baker) Cabrera & scr & - & - & $\mathrm{x}$ & - & - & - & - & - & - & - \\
\hline Eupatorium megaphyllum Baker & scr & - & $\mathrm{x}$ & - & - & - & - & - & - & - & - \\
\hline Eupatorium vauthierianum DC. & scr & - & $\mathrm{x}$ & - & - & - & - & - & - & - & - \\
\hline Heterocondylus vitalbae (DC.) R.M.King \& H.Rob. & twi & - & $\mathrm{x}$ & - & - & - & - & - & $\mathrm{x}$ & - & - \\
\hline Mikania biformis DC. & twi & $\mathrm{x}$ & - & - & - & - & - & - & - & - & - \\
\hline Mikania cordifolia (L.f.) Willd. & twi & - & - & - & - & - & $\mathrm{x}$ & - & - & - & - \\
\hline Mikania cynanchifolia Hook. \& Arn. ex Baker & twi & - & - & - & - & - & - & - & $\mathrm{x}$ & - & - \\
\hline Mikania glomerata Spreng. & twi & $\mathrm{x}$ & $\mathrm{x}$ & - & - & $\mathrm{x}$ & - & - & $\mathrm{x}$ & $\mathrm{x}$ & $\mathrm{x}$ \\
\hline Mikania hemisphaerica Sch.Bip. ex Baker & twi & - & - & - & - & - & - & - & - & $\mathrm{x}$ & - \\
\hline Mikania hirsutissima DC. & twi & - & - & $\mathrm{x}$ & - & - & - & - & $\mathrm{x}$ & - & - \\
\hline Mikania lundiana DC. & twi & - & - & - & - & - & - & - & - & - & $\mathrm{x}$ \\
\hline Mikania micrantha Kunth & twi & - & $\mathrm{x}$ & - & - & $\mathrm{x}$ & $\mathrm{x}$ & - & $\mathrm{x}$ & $\mathrm{x}$ & $\mathrm{x}$ \\
\hline Mikania pyramidata Donn.Sm. & twi & - & $\mathrm{x}$ & - & - & - & - & - & - & - & - \\
\hline Mikania ramosissima Gardner & twi & - & $\mathrm{x}$ & - & - & - & - & - & - & - & $\mathrm{x}$ \\
\hline Mikania salviaefolia Gardner & twi & - & - & $\mathrm{x}$ & - & - & - & - & - & - & - \\
\hline Mikania triangularis Baker & twi & - & $\mathrm{x}$ & - & - & - & - & - & $\mathrm{x}$ & $\mathrm{x}$ & - \\
\hline Mutisia coccinea A.St.-Hil. & twi & $\mathrm{x}$ & $\mathrm{x}$ & - & - & - & - & - & $\mathrm{x}$ & - & - \\
\hline Pseudogynoxys cumingii (Benth.) H.Rob. \& Cuatrec. & scr & - & - & - & - & - & - & - & - & $\mathrm{x}$ & $\mathrm{x}$ \\
\hline Trixis antimenorrhoea (Schrank) Kuntze & scr & - & - & - & - & - & - & - & $\mathrm{x}$ & $\mathrm{x}$ & - \\
\hline Trixis divaricata (Kunth) Spreng. & scr & - & $\mathrm{x}$ & - & - & - & - & - & - & - & - \\
\hline Vernonanthura crassa (Vell.) H.Rob. & scr & - & $\mathrm{x}$ & - & - & - & - & - & - & - & - \\
\hline Vernonanthura diffusa (Less.) H.Rob. & scr & - & $\mathrm{x}$ & - & - & - & - & - & - & - & - \\
\hline \multicolumn{12}{|l|}{ BASELLACEAE } \\
\hline Anredera cordifolia (Ten.) Steenis & twi & - & - & - & - & - & - & - & $\mathrm{x}$ & - & - \\
\hline \multicolumn{12}{|l|}{ BIGNONIACEAE } \\
\hline Adenocalymma bracteatum (Cham.) DC. & ten & - & $\mathrm{x}$ & - & $\mathrm{x}$ & $\mathrm{x}$ & $\mathrm{x}$ & $\mathrm{x}$ & $\mathrm{x}$ & - & $\mathrm{x}$ \\
\hline Adenocalymma dusenii Kraenzl. & ten & $\mathrm{x}$ & - & - & - & - & - & - & - & - & - \\
\hline Adenocalymma marginatum (Cham.) DC. & ten & - & $\mathrm{x}$ & - & - & $\mathrm{x}$ & - & - & $\mathrm{x}$ & $\mathrm{x}$ & $\mathrm{x}$ \\
\hline Adenocalymma paulistarum Bureau \& K.Schum. & ten & - & $\mathrm{x}$ & - & - & - & - & - & - & - & $\mathrm{x}$ \\
\hline Amphilophium paniculatum (L.) Kunth & ten & - & $\mathrm{x}$ & - & $\mathrm{x}$ & $\mathrm{x}$ & $\mathrm{x}$ & $\mathrm{x}$ & $\mathrm{x}$ & - & - \\
\hline $\begin{array}{l}\text { Anemopaegma chamberlaynii } \\
\text { (Sims) Bureau \& K.Schum. }\end{array}$ & ten & - & $\mathrm{x}$ & - & - & $\mathrm{x}$ & - & $\mathrm{x}$ & $\mathrm{x}$ & $\mathrm{x}$ & - \\
\hline Arrabidaea chica (Bonpl.) Verl. & ten & - & $\mathrm{x}$ & - & $\mathrm{x}$ & - & $\mathrm{x}$ & - & - & - & $\mathrm{x}$ \\
\hline Arrabidaea conjugata (Vell.) Mart. & ten & - & - & - & - & $\mathrm{x}$ & - & $\mathrm{x}$ & - & - & $\mathrm{x}$ \\
\hline Arrabidaea craterophora (DC.) Bureau & ten & - & - & $\mathrm{x}$ & - & - & $\mathrm{x}$ & - & - & - & - \\
\hline Arrabidaea florida DC. & ten & - & - & - & - & $\mathrm{x}$ & $\mathrm{x}$ & - & - & - & $\mathrm{x}$ \\
\hline Arrabidaea formosa (Bureau) Sandwith & ten & - & $\mathrm{x}$ & $\mathrm{x}$ & $\mathrm{x}$ & - & - & - & - & - & - \\
\hline Arrabidaea leucopogon (Cham.) Sandwith & ten & - & - & - & $\mathrm{x}$ & - & $\mathrm{x}$ & - & - & - & - \\
\hline Arrabidaea mutabilis Bureau \& K.Schum. & ten & - & - & - & - & - & - & $\mathrm{x}$ & - & - & - \\
\hline Arrabidaea pubescens (L.) A.H.Gentry & ten & - & $\mathrm{x}$ & - & - & - & - & - & - & - & - \\
\hline
\end{tabular}


Table 2. Continued...

\begin{tabular}{|c|c|c|c|c|c|c|c|c|c|c|c|}
\hline Family and species & Climb* & ANG & ARA & BH & PF & $\mathbf{R C}$ & $\mathbf{R P}$ & SC & SG & SV & VAS \\
\hline Arrabidaea pulchella (Cham.) Bureau & ten & $\mathrm{x}$ & $\mathrm{x}$ & - & $\mathrm{x}$ & - & - & - & - & - & $\mathrm{x}$ \\
\hline Arrabidaea pulchra (Cham.) Sandwith & ten & - & $\mathrm{x}$ & $\mathrm{x}$ & - & - & - & - & - & - & - \\
\hline Arrabidaea samydoides (Cham.) Sandwith & ten & $\mathrm{x}$ & $\mathrm{x}$ & $\mathrm{x}$ & - & - & - & - & $\mathrm{x}$ & - & - \\
\hline Arrabidaea selloi (Spreng.) Sandwith & ten & - & $\mathrm{x}$ & - & $\mathrm{x}$ & $\mathrm{x}$ & - & $\mathrm{x}$ & $\mathrm{x}$ & - & $\mathrm{x}$ \\
\hline Arrabidaea triplinervia (Mart. ex DC.) Baill. & ten & $\mathrm{x}$ & $\mathrm{x}$ & $\mathrm{x}$ & $\mathrm{x}$ & $\mathrm{x}$ & $\mathrm{x}$ & $\mathrm{x}$ & $\mathrm{x}$ & $\mathrm{x}$ & - \\
\hline Bignonia binata Thunb. & ten & - & - & - & - & - & - & - & - & - & $\mathrm{x}$ \\
\hline Bignonia campanulata Cham. & ten & - & $\mathrm{x}$ & - & - & $\mathrm{x}$ & - & $\mathrm{x}$ & $\mathrm{x}$ & $\mathrm{x}$ & $\mathrm{x}$ \\
\hline $\begin{array}{l}\text { Clytostoma sciuripabulum } \\
\text { (K.Schum.) Bureau \& K.Schum. }\end{array}$ & ten & - & $\mathrm{x}$ & - & - & - & - & - & - & - & $\mathrm{x}$ \\
\hline Cuspidaria convoluta (Vell.) A.H.Gentry & ten & - & $\mathrm{x}$ & - & - & - & - & - & $\mathrm{x}$ & - & - \\
\hline Cuspidaria floribunda (DC.) A.H.Gentry & ten & - & $\mathrm{x}$ & - & - & - & $\mathrm{x}$ & - & $\mathrm{x}$ & - & $\mathrm{x}$ \\
\hline Distictella elongata (Vahl) Urb. & ten & - & - & - & - & - & - & - & - & - & $\mathrm{x}$ \\
\hline Distictella granulosa (Klotzsch) Urb. & ten & - & - & - & - & - & - & - & - & $\mathrm{x}$ & - \\
\hline Distictella magnoliifolia (Kunth) Sandwith & ten & - & - & - & - & $\mathrm{x}$ & - & - & - & - & - \\
\hline Fridericia speciosa Mart. & ten & $\mathrm{x}$ & $\mathrm{x}$ & - & $\mathrm{x}$ & $\mathrm{x}$ & - & $\mathrm{x}$ & $\mathrm{x}$ & - & - \\
\hline Lundia obliqua Sond. & ten & $\mathrm{x}$ & $\mathrm{x}$ & - & $\mathrm{x}$ & $\mathrm{x}$ & $\mathrm{x}$ & $\mathrm{x}$ & $\mathrm{x}$ & $\mathrm{x}$ & $\mathrm{x}$ \\
\hline Lundia virginalis DC. & ten & - & - & - & - & - & - & - & $\mathrm{x}$ & - & - \\
\hline Macfadyena mollis (Sond.) Seem. & ten & - & $\mathrm{x}$ & - & - & - & - & - & - & - & $\mathrm{x}$ \\
\hline Macfadyena unguis-cati (L.) A.H.Gentry & ten & $\mathrm{x}$ & $\mathrm{x}$ & $\mathrm{x}$ & $\mathrm{x}$ & $\mathrm{x}$ & $\mathrm{x}$ & $\mathrm{x}$ & $\mathrm{x}$ & $\mathrm{x}$ & $\mathrm{x}$ \\
\hline Mansoa difficilis (Cham.) Bureau \& K.Schum. & ten & $\mathrm{x}$ & $\mathrm{x}$ & - & - & - & - & $\mathrm{x}$ & $\mathrm{x}$ & $\mathrm{x}$ & $\mathrm{x}$ \\
\hline Melloa quadrivalvis (Jacq.) A.H.Gentry & ten & - & $\mathrm{x}$ & - & - & - & - & $\mathrm{x}$ & - & $\mathrm{x}$ & - \\
\hline Paragonia pyramidata (Rich.) Bureau & ten & - & - & - & $\mathrm{x}$ & - & $\mathrm{x}$ & $\mathrm{x}$ & $\mathrm{x}$ & - & $\mathrm{x}$ \\
\hline Pithecoctenium crucigerum (L.) A.H.Gentry & ten & - & $\mathrm{x}$ & - & - & - & - & $\mathrm{x}$ & $\mathrm{x}$ & $\mathrm{x}$ & $\mathrm{x}$ \\
\hline Pleonotoma stichadenia K.Schum. & ten & - & - & $\mathrm{x}$ & - & - & - & - & - & - & - \\
\hline Pleonotoma tetraquetra (Cham.) Bureau & ten & - & $\mathrm{x}$ & - & $\mathrm{x}$ & - & - & - & $\mathrm{x}$ & - & $\mathrm{x}$ \\
\hline Pyrostegia venusta (Ker Gawl.) Miers & ten & $\mathrm{x}$ & $\mathrm{x}$ & $\mathrm{x}$ & $\mathrm{x}$ & - & $\mathrm{x}$ & $\mathrm{x}$ & $\mathrm{x}$ & $\mathrm{x}$ & $\mathrm{x}$ \\
\hline Stizophyllum perforatum (Cham.) Miers & ten & - & $\mathrm{x}$ & $\mathrm{x}$ & - & $\mathrm{x}$ & $\mathrm{x}$ & $\mathrm{x}$ & $\mathrm{x}$ & $\mathrm{x}$ & $\mathrm{x}$ \\
\hline Tynanthus cognatus (Cham.) Miers & ten & - & - & - & - & $\mathrm{x}$ & - & - & $\mathrm{x}$ & - & - \\
\hline Tynanthus elegans Miers & ten & - & $\mathrm{x}$ & - & $\mathrm{x}$ & - & - & $\mathrm{x}$ & - & - & - \\
\hline Tynanthus fasciculatus (Vell.) Miers & ten & - & $\mathrm{x}$ & - & - & - & - & - & $\mathrm{x}$ & - & $\mathrm{x}$ \\
\hline Tynanthus labiatus (Cham.) Miers & ten & - & - & - & - & $\mathrm{x}$ & - & - & - & - & - \\
\hline Tynanthus micranthus Corr.Méllo ex K.Schum. & ten & - & - & - & - & - & - & - & - & - & $\mathrm{x}$ \\
\hline \multicolumn{12}{|l|}{ BORAGINACEAE } \\
\hline Cordia polycephala (Lam.) I.M.Johnst. & scr & $\mathrm{x}$ & - & - & - & - & - & - & - & - & - \\
\hline Tournefortia bicolor $\mathrm{Sw}$. & scr & - & - & - & - & - & - & - & $\mathrm{x}$ & - & - \\
\hline Tournefortia elegans Cham. & scr & - & $\mathrm{x}$ & - & - & - & - & - & - & - & - \\
\hline Tournefortia paniculata Vent. & scr & - & $\mathrm{x}$ & - & - & - & $\mathrm{x}$ & - & - & - & - \\
\hline Tournefortia rubicunda Salzm. ex DC. & scr & - & - & - & - & $\mathrm{x}$ & $\mathrm{x}$ & - & - & - & - \\
\hline Tournefortia villosa Salzm. ex DC. & scr & - & - & - & - & - & - & - & $\mathrm{x}$ & - & - \\
\hline \multicolumn{12}{|l|}{ CACTACEAE } \\
\hline Pereskia aculeata Mill. & scr & - & $\mathrm{x}$ & - & - & $\mathrm{x}$ & - & $\mathrm{x}$ & $\mathrm{x}$ & $\mathrm{x}$ & $\mathrm{x}$ \\
\hline \multicolumn{12}{|l|}{ CAMPANULACEAE } \\
\hline $\begin{array}{l}\text { Centropogon argutus E.Wimm. } \\
\text { Cannabaceae }\end{array}$ & scr & - & - & - & $\mathrm{x}$ & - & - & - & - & - & - \\
\hline Celtis iguanaea (Jacq.) Sarg. & scr & - & $\mathrm{x}$ & - & $\mathrm{x}$ & - & - & - & - & - & $\mathrm{x}$ \\
\hline \multicolumn{12}{|l|}{ CELASTRACEAE } \\
\hline Anthodon decussatum Ruiz \& Pav. & ten & - & $\mathrm{x}$ & - & $\mathrm{x}$ & $\mathrm{x}$ & - & - & - & - & $\mathrm{x}$ \\
\hline Cheiloclinium cognatum (Miers) A.C.Sm. & ten & - & - & - & $\mathrm{x}$ & - & - & - & - & - & - \\
\hline Hippocratea volubilis $\mathrm{L}$. & ten & $\mathrm{x}$ & $\mathrm{x}$ & $\mathrm{x}$ & - & $\mathrm{x}$ & $\mathrm{x}$ & $\mathrm{x}$ & $\mathrm{x}$ & $\mathrm{x}$ & - \\
\hline Peritassa calypsoides (Cambess.) A.C.Sm. & scr & - & - & - & - & - & - & - & $\mathrm{x}$ & - & - \\
\hline Pristimera andina Miers & twi & - & - & - & - & $\mathrm{x}$ & - & $\mathrm{x}$ & $\mathrm{x}$ & - & - \\
\hline Semialarium paniculatum (Mart. ex Schult.) N.Hallé & scr & - & - & - & - & - & - & $\mathrm{x}$ & - & - & $\mathrm{x}$ \\
\hline
\end{tabular}


Table 2. Continued...

\begin{tabular}{|c|c|c|c|c|c|c|c|c|c|c|c|}
\hline Family and species & Climb* & ANG & ARA & BH & $\mathbf{P F}$ & RC & $\mathbf{R P}$ & SC & SG & SV & VAS \\
\hline \multicolumn{12}{|l|}{ COMBRETACEAE } \\
\hline Combretum discolor Taub. & twi & - & - & - & $\mathrm{x}$ & - & - & - & - & - & - \\
\hline \multicolumn{12}{|l|}{ COMMELINACEAE } \\
\hline Dichorisandra hexandra (Aubl.) Standl. & scr & - & $\mathrm{x}$ & - & - & - & - & - & - & - & - \\
\hline \multicolumn{12}{|l|}{ CONVOLVULACEAE } \\
\hline Bonamia burchellii (Choisy) Hallier & twi & - & - & - & - & - & $\mathrm{x}$ & - & - & - & - \\
\hline Ipomoea acuminata (Vahl) Roem. \& Schult. & twi & - & - & - & - & - & - & - & $\mathrm{x}$ & - & - \\
\hline Ipomoea alba $\mathrm{L}$. & twi & - & - & - & - & - & - & - & - & - & $\mathrm{x}$ \\
\hline Ipomoea brasiliana Meisn. & twi & - & $\mathrm{x}$ & - & - & - & - & - & - & - & - \\
\hline Ipomoea cairica (L.) Sweet & twi & - & - & - & - & $\mathrm{x}$ & - & - & $\mathrm{x}$ & $\mathrm{x}$ & - \\
\hline Ipomoea coccinea $\mathrm{L}$. & twi & - & - & - & - & $\mathrm{x}$ & - & - & - & - & - \\
\hline Ipomoea hederifolia $\mathrm{L}$. & twi & - & $\mathrm{x}$ & - & $\mathrm{x}$ & - & $\mathrm{x}$ & - & $\mathrm{x}$ & - & $\mathrm{x}$ \\
\hline Ipomoea nil (L.) Roth & twi & - & $\mathrm{x}$ & - & - & - & - & - & - & - & - \\
\hline Ipomoea pes-caprae (L.) R.Br. & twi & - & - & - & - & - & - & - & - & - & $\mathrm{x}$ \\
\hline Ipomoea purpurea (L.) Roth & twi & $\mathrm{x}$ & - & - & $\mathrm{x}$ & - & - & - & - & - & - \\
\hline Ipomoea quamoclit $\mathrm{L}$. & twi & - & $\mathrm{x}$ & - & - & - & - & - & $\mathrm{x}$ & - & $\mathrm{x}$ \\
\hline Ipomoea saopaulista O’Donell & twi & - & $\mathrm{x}$ & - & - & $\mathrm{x}$ & - & - & - & - & - \\
\hline Ipomoea syringifolia Meisn. & twi & - & $\mathrm{x}$ & - & - & - & - & - & - & - & - \\
\hline Ipomoea tubata Nees & twi & - & - & - & - & - & - & - & - & - & $\mathrm{x}$ \\
\hline Jacquemontia ciliata Sandwith & twi & - & $\mathrm{x}$ & - & - & - & - & - & - & - & - \\
\hline Jacquemontia densiflora (Meisn.) Hallier f. & twi & - & - & - & $\mathrm{x}$ & - & $\mathrm{x}$ & - & - & - & - \\
\hline Jacquemontia evolvuloides Meisn. & twi & - & - & - & - & - & $\mathrm{x}$ & - & - & - & - \\
\hline Jacquemontia velutina Choisy & twi & - & - & - & - & - & $\mathrm{x}$ & - & - & - & - \\
\hline Merremia aegyptia (L.) Urb. & twi & - & - & - & $\mathrm{x}$ & - & - & - & - & - & - \\
\hline Merremia cissoides (Lam.) Hallier f. & twi & - & - & - & - & - & $\mathrm{x}$ & - & - & $\mathrm{x}$ & - \\
\hline Merremia macrocalyx (Ruiz \& Pav.) O’Donell & twi & $\mathrm{x}$ & $\mathrm{x}$ & $\mathrm{x}$ & $\mathrm{x}$ & $\mathrm{x}$ & $\mathrm{x}$ & - & $\mathrm{x}$ & - & $\mathrm{x}$ \\
\hline \multicolumn{12}{|l|}{ CUCURBITACEAE } \\
\hline Cayaponia espelina (Silva Manso) Cogn. & ten & - & - & - & $\mathrm{x}$ & - & - & - & - & - & - \\
\hline Cayaponia tayuya (Vell.) Cogn. & ten & - & - & - & $\mathrm{x}$ & $\mathrm{x}$ & $\mathrm{x}$ & - & - & - & - \\
\hline Ceratosanthes hilariana Cogn. & ten & - & - & - & $\mathrm{x}$ & - & - & - & - & - & - \\
\hline Luffa cylindrica M.Roem. & ten & - & - & - & - & - & - & - & - & - & $\mathrm{x}$ \\
\hline Melothria cucumis Vell. & ten & - & - & - & - & - & - & - & - & $\mathrm{x}$ & $\mathrm{x}$ \\
\hline Melothria fluminensis Gardner & ten & - & - & - & - & - & $\mathrm{x}$ & - & $\mathrm{x}$ & - & - \\
\hline Melothria warmingii Cogn. & ten & - & - & - & - & - & $\mathrm{x}$ & - & - & - & - \\
\hline Momordica charantia $\mathrm{L}$. & ten & - & $\mathrm{x}$ & - & $\mathrm{x}$ & - & $\mathrm{x}$ & - & $\mathrm{x}$ & - & $\mathrm{x}$ \\
\hline Psiguria ternata (M. Roem.) C.Jeffrey & ten & - & $\mathrm{x}$ & - & - & - & - & - & - & $\mathrm{x}$ & $\mathrm{x}$ \\
\hline Psiguria triphylla (Miq.) C.Jeffrey & ten & - & - & - & $\mathrm{x}$ & - & $\mathrm{x}$ & - & - & - & - \\
\hline Psiguria warmingiana (Cogn.) C.Jeffrey & ten & - & $\mathrm{x}$ & - & - & - & - & - & - & - & - \\
\hline Wilbrandia hibiscoides Silva Manso & ten & - & - & - & - & - & - & - & $\mathrm{x}$ & - & - \\
\hline Wilbrandia longibracteata Cogn. & ten & - & $\mathrm{x}$ & - & - & - & - & - & - & - & - \\
\hline Wilbrandia verticillata Cogn. & ten & - & - & - & $\mathrm{x}$ & - & - & - & - & $\mathrm{x}$ & - \\
\hline \multicolumn{12}{|l|}{ DILLENIACEAE } \\
\hline Davilla rugosa Poir. & twi & - & $\mathrm{x}$ & $\mathrm{x}$ & - & - & - & $\mathrm{x}$ & $\mathrm{x}$ & - & $\mathrm{x}$ \\
\hline Doliocarpus dentatus (Aubl.) Standl. & twi & - & - & $\mathrm{x}$ & - & - & $\mathrm{x}$ & - & - & - & - \\
\hline Tetracera oblongata DC. & twi & $\mathrm{x}$ & - & - & - & - & - & - & - & - & - \\
\hline Tetracera willdenowiana Steud. & twi & - & - & - & - & - & - & - & - & - & $\mathrm{x}$ \\
\hline \multicolumn{12}{|l|}{ DIOSCOREACEAE } \\
\hline Dioscorea altissima Lam. & twi & - & - & - & - & - & - & - & - & $\mathrm{x}$ & - \\
\hline Dioscorea dodecaneura Vell. & twi & - & $\mathrm{x}$ & - & - & - & $\mathrm{x}$ & - & - & - & - \\
\hline Dioscorea macrocapsa R.Knuth & twi & - & - & - & - & - & - & - & $\mathrm{x}$ & - & - \\
\hline Dioscorea macrocarpa Uline & twi & - & - & - & - & - & - & - & $\mathrm{x}$ & - & - \\
\hline
\end{tabular}


Table 2. Continued...

\begin{tabular}{|c|c|c|c|c|c|c|c|c|c|c|c|}
\hline Family and species & Climb* & ANG & ARA & BH & $\mathbf{P F}$ & $\mathbf{R C}$ & $\mathbf{R P}$ & SC & SG & SV & VAS \\
\hline Dioscorea monandra Hauman & twi & - & - & - & $\mathrm{x}$ & - & - & - & - & - & $\mathrm{x}$ \\
\hline Dioscorea multiflora Griseb. & twi & - & - & - & - & $\mathrm{x}$ & $\mathrm{x}$ & - & - & $\mathrm{x}$ & $\mathrm{x}$ \\
\hline $\begin{array}{l}\text { Dioscorea pseudomacrocapsa } \\
\text { G.M.Barroso, E.F.Guim. \& Sucre }\end{array}$ & twi & - & - & - & - & - & - & - & $\mathrm{x}$ & - & - \\
\hline \multicolumn{12}{|l|}{ EUPHORBIACEAE } \\
\hline Bia alienata Didr. & twi & - & $\mathrm{x}$ & - & - & - & - & - & $\mathrm{x}$ & - & - \\
\hline Dalechampia olfersiana Müll.Arg. & twi & - & - & - & - & - & - & - & $\mathrm{x}$ & - & - \\
\hline Dalechampia pentaphylla Lam. & twi & - & - & - & $\mathrm{x}$ & - & $\mathrm{x}$ & - & $\mathrm{x}$ & $\mathrm{x}$ & $\mathrm{x}$ \\
\hline Dalechampia scandens L. & twi & - & $\mathrm{x}$ & - & - & - & $\mathrm{x}$ & - & - & - & - \\
\hline Dalechampia stipulacea Müll.Arg. & twi & - & $\mathrm{x}$ & - & - & - & - & - & $\mathrm{x}$ & - & $\mathrm{x}$ \\
\hline Dalechampia triphylla Lam. & twi & - & $\mathrm{x}$ & - & - & $\mathrm{x}$ & $\mathrm{x}$ & - & $\mathrm{x}$ & - & - \\
\hline Romanoa tamnoides (A.Juss.) Radcl.-Sm. & twi & - & - & - & $\mathrm{x}$ & - & - & - & - & - & - \\
\hline Tragia volubilis $\mathrm{L}$. & twi & - & - & - & - & $\mathrm{x}$ & - & - & - & $\mathrm{x}$ & - \\
\hline \multicolumn{12}{|l|}{ FABACEAE } \\
\hline Acacia adhaerens Benth. & scr & - & - & - & - & - & - & - & - & $\mathrm{x}$ & - \\
\hline Acacia paniculata Willd. & twi & - & $\mathrm{x}$ & $\mathrm{x}$ & - & - & - & $\mathrm{x}$ & - & - & $\mathrm{x}$ \\
\hline Acacia plumosa Mart. ex Colla & $\mathrm{scr}$ & - & - & - & - & - & - & $\mathrm{x}$ & - & - & - \\
\hline Bauhinia leiopetala Benth. & ten & - & - & $\mathrm{x}$ & - & - & - & - & - & - & - \\
\hline Bauhinia microstachya (Raddi) J.F.Macbr. & ten & - & - & - & $\mathrm{x}$ & $\mathrm{x}$ & - & $\mathrm{x}$ & - & $\mathrm{x}$ & - \\
\hline Bauhinia siqueiraei Ducke & ten & - & - & - & - & - & - & - & - & - & $\mathrm{x}$ \\
\hline Calopogonium caeruleum (Benth.) C.Wright & twi & - & - & - & $\mathrm{x}$ & - & - & - & - & - & - \\
\hline Calopogonium mucunoides Desv. & twi & - & - & - & $\mathrm{x}$ & - & $\mathrm{x}$ & - & - & - & - \\
\hline Camptosema ellipticum (Desv.) Burkart & twi & - & - & - & - & - & $\mathrm{x}$ & - & - & - & - \\
\hline Canavalia grandiflora Benth. & twi & - & - & - & - & $\mathrm{x}$ & $\mathrm{x}$ & - & - & - & - \\
\hline Canavalia palmeri (Piper) Standl. & twi & - & - & - & $\mathrm{x}$ & - & - & - & - & - & - \\
\hline Canavalia parviflora Benth. & twi & - & - & - & - & - & - & - & $\mathrm{x}$ & - & - \\
\hline Canavalia picta Mart. ex Benth. & twi & - & - & - & - & - & - & - & $\mathrm{x}$ & $\mathrm{x}$ & - \\
\hline Centrosema arenarium Benth. & twi & - & - & - & $\mathrm{x}$ & - & - & - & - & - & - \\
\hline Centrosema grandiflorum Benth. & twi & - & - & - & $\mathrm{x}$ & - & - & - & - & - & - \\
\hline Centrosema pascuorum Mart. ex Benth. & twi & - & - & - & $\mathrm{x}$ & - & - & - & - & - & - \\
\hline Centrosema plumieri (Turpin ex Pers.) Benth. & twi & - & - & - & $\mathrm{x}$ & - & - & - & - & - & - \\
\hline Centrosema pubescens Benth. & twi & $\mathrm{x}$ & - & - & $\mathrm{x}$ & - & $\mathrm{x}$ & - & - & - & - \\
\hline $\begin{array}{l}\text { Centrosema sagittatum } \\
\text { (Humb. \& Bonpl. ex Willd.) Brandegee }\end{array}$ & twi & - & $\mathrm{x}$ & - & $\mathrm{x}$ & $\mathrm{x}$ & $\mathrm{x}$ & - & - & - & - \\
\hline Centrosema venosum Mart. ex Benth. & twi & - & - & - & $\mathrm{x}$ & - & - & - & - & - & - \\
\hline Centrosema vexillatum Benth. & twi & - & - & - & $\mathrm{x}$ & - & - & - & - & - & - \\
\hline Centrosema virginianum (L.) Benth. & twi & $\mathrm{x}$ & - & - & $\mathrm{x}$ & - & - & - & - & - & - \\
\hline Chaetocalyx brasiliensis (Vogel) Benth. & twi & - & - & - & $\mathrm{x}$ & - & - & - & - & - & - \\
\hline Clitoria falcata Lam. & twi & - & - & - & - & - & $\mathrm{x}$ & - & - & - & - \\
\hline Dalbergia frutescens (Vell.) Britton & scr & - & - & - & - & - & - & $\mathrm{x}$ & $\mathrm{x}$ & - & - \\
\hline Dioclea latifolia Benth. & twi & - & - & - & - & $\mathrm{x}$ & - & - & - & - & - \\
\hline Dioclea rufescens Benth. & twi & - & - & - & - & - & - & - & $\mathrm{x}$ & - & - \\
\hline Dioclea violacea Mart. ex Benth. & twi & - & - & - & - & - & - & - & $\mathrm{x}$ & - & - \\
\hline Dioclea virgata (Rich.) Amshoff & twi & - & - & - & $\mathrm{x}$ & - & - & - & - & - & - \\
\hline Galactia striata (Jacq.) Urb. & twi & - & - & - & - & - & - & - & - & $\mathrm{x}$ & - \\
\hline Machaerium dimorphandrum Hoehne & scr & - & - & - & - & - & - & $\mathrm{x}$ & - & - & - \\
\hline Machaerium oblongifolium Vogel & scr & - & $\mathrm{x}$ & - & $\mathrm{x}$ & - & - & - & - & - & $\mathrm{x}$ \\
\hline Machaerium uncinatum (Vell.) Benth. & scr & - & - & $\mathrm{x}$ & - & - & - & - & - & - & - \\
\hline Macroptilium atropurpureum (DC.) Urb. & twi & - & - & - & - & $\mathrm{x}$ & $\mathrm{x}$ & - & - & - & - \\
\hline $\begin{array}{l}\text { Macroptilium bracteatum (Nees \& C. Mart.) } \\
\text { Maréchal \& Baudet }\end{array}$ & twi & - & - & - & - & - & $\mathrm{x}$ & - & - & - & - \\
\hline
\end{tabular}


Table 2. Continued...

\begin{tabular}{|c|c|c|c|c|c|c|c|c|c|c|c|}
\hline Family and species & Climb* & ANG & ARA & BH & PF & RC & $\mathbf{R P}$ & SC & SG & SV & VAS \\
\hline Mucuna pruriens (L.) DC. & twi & - & - & - & $\mathrm{x}$ & - & $\mathrm{x}$ & - & - & - & - \\
\hline Phaseolus lunatus L. & twi & - & - & - & $\mathrm{x}$ & - & - & - & - & - & - \\
\hline Rhynchosia phaseoloides (Sw.) DC. & twi & - & - & - & $\mathrm{x}$ & $\mathrm{x}$ & $\mathrm{x}$ & - & $\mathrm{x}$ & - & - \\
\hline Teramnus uncinatus (L.) Sw. & twi & - & - & - & - & - & $\mathrm{x}$ & - & - & $\mathrm{x}$ & - \\
\hline $\begin{array}{l}\text { Vigna candida (Vell.) } \\
\text { Maréchal, Mascherpa \& Stainier }\end{array}$ & twi & - & - & - & - & - & - & - & $\mathrm{x}$ & $\mathrm{x}$ & - \\
\hline Vigna caracalla (L.) Verdc. & twi & - & - & - & $\mathrm{x}$ & - & - & - & - & - & - \\
\hline Vigna peduncularis (Kunth) Fawc. \& Rendle & twi & - & - & - & $\mathrm{x}$ & - & - & - & - & - & - \\
\hline \multicolumn{12}{|l|}{ LOGANIACEAE } \\
\hline Strychnos brasiliensis (Spreng.) Mart. & $\mathrm{scr}$ & - & - & $\mathrm{x}$ & - & - & - & - & - & - & - \\
\hline \multicolumn{12}{|l|}{ MALPIGHIACEAE } \\
\hline Banisteriopsis adenopoda (A.Juss.) B.Gates & twi & $\mathrm{x}$ & $\mathrm{x}$ & $\mathrm{x}$ & - & - & - & - & $\mathrm{x}$ & - & $\mathrm{x}$ \\
\hline Banisteriopsis anisandra (A.Juss.) B.Gates & twi & - & - & $\mathrm{x}$ & - & - & - & - & $\mathrm{x}$ & $\mathrm{x}$ & - \\
\hline Banisteriopsis argyrophylla (A.Juss.) B.Gates & twi & - & - & $\mathrm{x}$ & - & - & $\mathrm{x}$ & - & $\mathrm{x}$ & - & - \\
\hline Banisteriopsis lutea (Griseb.) Cuatrec. & twi & - & $\mathrm{x}$ & - & $\mathrm{x}$ & - & - & - & $\mathrm{x}$ & - & $\mathrm{x}$ \\
\hline Banisteriopsis muricata (Cav.) Cuatrec. & twi & - & - & - & - & $\mathrm{x}$ & $\mathrm{x}$ & - & $\mathrm{x}$ & - & - \\
\hline $\begin{array}{l}\text { Banisteriopsis nitrosiodora (Griseb.) } \\
\text { O’Donell \& Lourteig }\end{array}$ & twi & - & - & - & - & - & $\mathrm{x}$ & - & - & - & - \\
\hline Banisteriopsis oxyclada (A.Juss.) B.Gates & twi & $\mathrm{x}$ & $\mathrm{x}$ & $\mathrm{x}$ & $\mathrm{x}$ & - & $\mathrm{x}$ & - & - & $\mathrm{x}$ & $\mathrm{x}$ \\
\hline Banisteriopsis pubipetala (A.Juss.) Cuatrec. & twi & - & - & $\mathrm{x}$ & - & - & $\mathrm{x}$ & - & $\mathrm{x}$ & - & - \\
\hline Dicella bracteosa (A.Juss.) Griseb. & twi & - & $\mathrm{x}$ & - & - & $\mathrm{x}$ & - & - & $\mathrm{x}$ & $\mathrm{x}$ & $\mathrm{x}$ \\
\hline Heteropterys aceroides Griseb. & twi & - & - & - & $\mathrm{x}$ & - & - & - & $\mathrm{x}$ & - & - \\
\hline Heteropterys acutifolia A.Juss. & twi & - & - & - & - & - & - & - & $\mathrm{x}$ & - & - \\
\hline Heteropterys argyrophaea A.Juss. & twi & - & - & - & $\mathrm{x}$ & - & - & - & - & - & - \\
\hline Heteropterys bicolor A.Juss. & twi & - & - & - & - & - & - & - & $\mathrm{x}$ & - & - \\
\hline Heteropterys byrsonimifolia A.Juss. & twi & - & $\mathrm{x}$ & - & - & - & - & - & - & - & - \\
\hline Heteropterys campestris A.Juss. & twi & - & - & $\mathrm{x}$ & - & - & - & - & - & - & - \\
\hline Heteropterys dumetorum (Griseb.) Nied. & twi & - & - & - & $\mathrm{x}$ & - & $\mathrm{x}$ & - & - & - & - \\
\hline Heteropterys escalloniifolia A.Juss. & twi & - & - & $\mathrm{x}$ & $\mathrm{x}$ & - & - & - & - & - & - \\
\hline Heteropterys intermedia (A.Juss.) Griseb. & twi & - & $\mathrm{x}$ & - & - & - & - & - & - & - & - \\
\hline Heteropterys pauciflora A.Juss. & twi & - & - & - & - & - & - & $\mathrm{x}$ & $\mathrm{x}$ & - & $\mathrm{x}$ \\
\hline Janusia guaranitica (A.St.-Hil.) A.Juss. & twi & - & - & - & - & - & $\mathrm{x}$ & - & - & - & - \\
\hline Mascagnia anisopetala (A.Juss.) Griseb. & twi & - & - & - & - & - & - & - & $\mathrm{x}$ & $\mathrm{x}$ & $\mathrm{x}$ \\
\hline Mascagnia chlorocarpa (A.Juss.) Griseb. & twi & - & - & - & $\mathrm{x}$ & - & $\mathrm{x}$ & $\mathrm{x}$ & - & - & $\mathrm{x}$ \\
\hline Mascagnia cordifolia (A.Juss.) Griseb. & twi & - & $\mathrm{x}$ & $\mathrm{x}$ & - & $\mathrm{x}$ & $\mathrm{x}$ & - & $\mathrm{x}$ & - & $\mathrm{x}$ \\
\hline Mascagnia lasiandra (A.Juss.) Nied. & twi & - & $\mathrm{x}$ & - & - & - & - & - & - & - & - \\
\hline Mascagnia sepium (A.Juss.) Griseb. & twi & - & - & - & - & - & - & - & $\mathrm{x}$ & - & - \\
\hline Peixotoa paludosa Turcz. & twi & - & - & $\mathrm{x}$ & - & - & - & - & - & - & - \\
\hline Peixotoa reticulata Griseb. & twi & - & - & - & - & - & $\mathrm{x}$ & - & - & - & - \\
\hline Stigmaphyllon lalandianum A.Juss. & twi & - & $\mathrm{x}$ & - & - & - & - & $\mathrm{x}$ & $\mathrm{x}$ & - & $\mathrm{x}$ \\
\hline Stigmaphyllon puberulum Griseb. & twi & - & - & - & $\mathrm{x}$ & - & - & - & - & - & - \\
\hline Stigmaphyllon tomentosum A.Juss. & twi & - & - & - & - & - & - & - & - & - & $\mathrm{x}$ \\
\hline Tetrapterys chamaecerasifolia A.Juss. & twi & - & - & $\mathrm{x}$ & - & - & - & - & - & - & - \\
\hline Tetrapterys guilleminiana A.Juss. & twi & - & - & - & - & - & - & $\mathrm{x}$ & $\mathrm{x}$ & $\mathrm{x}$ & $\mathrm{x}$ \\
\hline Tetrapterys multiglandulosa A.Juss. & twi & $\mathrm{x}$ & - & - & - & - & - & - & - & - & $\mathrm{x}$ \\
\hline Tetrapterys phlomoides (Spreng.) Nied. & twi & - & $\mathrm{x}$ & - & - & - & - & - & - & - & - \\
\hline Tetrapterys ramiflora A.Juss. & twi & - & - & - & - & - & - & - & - & - & $\mathrm{x}$ \\
\hline Tetrapterys xylosteifolia A.Juss. & twi & - & - & - & - & - & - & - & $\mathrm{x}$ & - & - \\
\hline \multicolumn{12}{|l|}{ MALVACEAE } \\
\hline Byttneria australis A.St.-Hil. & scr & - & $\mathrm{x}$ & - & - & - & - & - & - & - & - \\
\hline Byttneria catalpifolia Jacq. & scr & - & $\mathrm{x}$ & - & - & - & - & - & $\mathrm{x}$ & - & $\mathrm{x}$ \\
\hline
\end{tabular}


Table 2. Continued...

\begin{tabular}{|c|c|c|c|c|c|c|c|c|c|c|c|}
\hline Family and species & Climb* & ANG & ARA & BH & $\mathbf{P F}$ & $\mathbf{R C}$ & $\mathbf{R P}$ & $\mathrm{SC}$ & SG & SV & VAS \\
\hline Byttneria laevigata Schott ex Pohl & scr & - & - & $\mathrm{x}$ & - & - & - & - & - & - & - \\
\hline \multicolumn{12}{|l|}{ MARCGRAVIACEAE } \\
\hline Marcgravia polyantha Delpino & scr & - & - & - & - & $\mathrm{x}$ & - & - & - & - & - \\
\hline \multicolumn{12}{|l|}{ MENISPERMACEAE } \\
\hline Cissampelos andromorpha DC. & twi & - & $\mathrm{x}$ & - & $\mathrm{x}$ & - & - & - & - & - & - \\
\hline Cissampelos glaberrima A.St.-Hil. & twi & - & $\mathrm{x}$ & - & $\mathrm{x}$ & $\mathrm{x}$ & $\mathrm{x}$ & - & $\mathrm{x}$ & $\mathrm{x}$ & - \\
\hline Cissampelos pareira $\mathrm{L}$. & twi & - & $\mathrm{x}$ & - & $\mathrm{x}$ & - & $\mathrm{x}$ & - & - & - & $\mathrm{x}$ \\
\hline Odontocarya acuparata Miers & twi & - & $\mathrm{x}$ & - & - & - & - & - & - & - & - \\
\hline \multicolumn{12}{|l|}{ NYCTAGINACEAE } \\
\hline Bougainvillea spectabilis Willd. & $\mathrm{scr}$ & - & - & $\mathrm{x}$ & - & - & - & - & - & - & - \\
\hline Pisonia aculeata $\mathrm{L}$. & scr & - & - & - & - & - & - & - & $\mathrm{x}$ & - & - \\
\hline \multicolumn{12}{|l|}{ PASSIFLORACEAE } \\
\hline Passiflora amethystina J.C.Mikan & ten & - & $\mathrm{x}$ & - & $\mathrm{x}$ & - & - & - & $\mathrm{x}$ & - & - \\
\hline Passiflora capsularis L. & ten & $\mathrm{x}$ & $\mathrm{x}$ & - & - & $\mathrm{x}$ & - & - & - & $\mathrm{x}$ & - \\
\hline Passiflora cincinnata Mast. & ten & - & - & - & - & - & $\mathrm{x}$ & - & - & - & - \\
\hline Passiflora foetida $\mathrm{L}$. & ten & - & - & - & - & $\mathrm{x}$ & $\mathrm{x}$ & - & - & - & - \\
\hline Passiflora galbana Mast. & ten & - & - & $\mathrm{x}$ & - & - & - & - & - & - & - \\
\hline Passiflora miersii Mart. & ten & - & - & - & - & - & $\mathrm{x}$ & - & $\mathrm{x}$ & - & - \\
\hline Passiflora sidiifolia M.Roem. & ten & - & - & - & - & $\mathrm{x}$ & - & - & - & $\mathrm{x}$ & - \\
\hline Passiflora suberosa $\mathrm{L}$. & ten & - & - & - & - & $\mathrm{x}$ & $\mathrm{x}$ & - & $\mathrm{x}$ & $\mathrm{x}$ & - \\
\hline Passiflora tricuspis Mast. & ten & - & - & - & $\mathrm{x}$ & - & $\mathrm{x}$ & - & - & - & - \\
\hline Passiflora vespertilio $\mathrm{L}$. & ten & - & - & - & - & - & - & - & $\mathrm{x}$ & - & - \\
\hline \multicolumn{12}{|l|}{ PIPERACEAE } \\
\hline Sarcorhachis obtusa (Miq.) Trel. & twi & - & - & - & - & - & - & - & - & $\mathrm{x}$ & - \\
\hline \multicolumn{12}{|l|}{ POLYGALACEAE } \\
\hline Bredemeyera floribunda Willd. & $\mathrm{scr}$ & $\mathrm{x}$ & $\mathrm{x}$ & $\mathrm{x}$ & $\mathrm{x}$ & - & $\mathrm{x}$ & - & - & - & - \\
\hline Diclidanthera laurifolia Mart. & twi & - & $\mathrm{x}$ & - & - & $\mathrm{x}$ & - & - & $\mathrm{x}$ & - & - \\
\hline Securidaca sellowiana Klotzsch & ten & $\mathrm{x}$ & - & - & $\mathrm{x}$ & - & - & - & - & - & - \\
\hline \multicolumn{12}{|l|}{ RANUNCULACEAE } \\
\hline Clematis dioica $\mathrm{L}$. & twi & $\mathrm{x}$ & $\mathrm{x}$ & $\mathrm{x}$ & - & $\mathrm{x}$ & - & - & $\mathrm{x}$ & $\mathrm{x}$ & $\mathrm{x}$ \\
\hline Gouania latifolia Reissek & ten & - & $\mathrm{x}$ & - & - & - & - & - & $\mathrm{x}$ & - & - \\
\hline Gouania ulmifolia Hook. \& Arn. & ten & $\mathrm{x}$ & - & - & - & - & $\mathrm{x}$ & - & - & - & - \\
\hline Gouania virgata Reissek & ten & - & $\mathrm{x}$ & - & $\mathrm{x}$ & $\mathrm{x}$ & $\mathrm{x}$ & - & $\mathrm{x}$ & $\mathrm{x}$ & $\mathrm{x}$ \\
\hline \multicolumn{12}{|l|}{ ROSACEAE } \\
\hline Rubus brasiliensis Mart. & $\mathrm{scr}$ & - & - & - & - & - & - & - & - & $\mathrm{x}$ & - \\
\hline Rubus urticifolius Poir. & scr & - & $\mathrm{x}$ & - & - & - & - & - & - & - & - \\
\hline \multicolumn{12}{|l|}{ RUBIACEAE } \\
\hline Chiococca alba (L.) Hitchc. & scr & - & $\mathrm{x}$ & $\mathrm{x}$ & $\mathrm{x}$ & $\mathrm{x}$ & $\mathrm{x}$ & - & $\mathrm{x}$ & $\mathrm{x}$ & - \\
\hline Coccocypselum lanceolatum (Ruiz \& Pav.) Pers. & scr & $\mathrm{x}$ & - & - & - & - & - & - & - & - & - \\
\hline Coutarea hexandra (Jacq.) K.Schum. & scr & - & - & - & - & $\mathrm{x}$ & - & - & - & - & - \\
\hline Manettia cordifolia Mart. & twi & $\mathrm{x}$ & $\mathrm{x}$ & - & $\mathrm{x}$ & $\mathrm{x}$ & $\mathrm{x}$ & - & $\mathrm{x}$ & - & $\mathrm{x}$ \\
\hline Manettia luteorubra (Vell.) Benth. & twi & - & - & - & - & - & - & - & - & - & $\mathrm{x}$ \\
\hline \multicolumn{12}{|l|}{ SAPINDACEAE } \\
\hline Cardiospermum corindum $\mathrm{L}$. & ten & - & - & - & - & - & $\mathrm{x}$ & - & - & - & - \\
\hline Cardiospermum grandiflorum Sw. & ten & - & $\mathrm{x}$ & - & $\mathrm{x}$ & - & $\mathrm{x}$ & - & $\mathrm{x}$ & $\mathrm{x}$ & - \\
\hline Paullinia elegans Cambess. & ten & - & $\mathrm{x}$ & - & - & - & $\mathrm{x}$ & - & - & - & $\mathrm{x}$ \\
\hline Paullinia firma Radlk. & ten & - & - & - & $\mathrm{x}$ & - & - & - & - & - & - \\
\hline Paullinia meliifolia Juss. & ten & $\mathrm{x}$ & - & - & - & - & - & - & - & $\mathrm{x}$ & $\mathrm{x}$ \\
\hline Paullinia pinnata $\mathrm{L}$. & ten & - & - & - & - & - & - & - & $\mathrm{x}$ & - & - \\
\hline Paullinia rhomboidea Radlk. & ten & - & - & - & - & $\mathrm{x}$ & - & - & $\mathrm{x}$ & - & $\mathrm{x}$ \\
\hline Paullinia seminuda Radlk. & ten & - & - & - & - & $\mathrm{x}$ & - & - & - & - & - \\
\hline
\end{tabular}


Table 2. Continued...

\begin{tabular}{|c|c|c|c|c|c|c|c|c|c|c|c|}
\hline Family and species & Climb* & ANG & ARA & $\mathbf{B H}$ & PF & $\mathbf{R C}$ & $\mathbf{R P}$ & SC & SG & SV & VAS \\
\hline Paullinia spicata Benth. & ten & - & $\mathrm{x}$ & - & - & $\mathrm{x}$ & $\mathrm{x}$ & - & - & - & $\mathrm{x}$ \\
\hline Paullinia trigonia Vell. & ten & - & - & - & - & - & - & - & - & $\mathrm{x}$ & - \\
\hline Serjania caracasana (Jacq.) Willd. & ten & - & $\mathrm{x}$ & - & $\mathrm{x}$ & $\mathrm{x}$ & $\mathrm{x}$ & $\mathrm{x}$ & $\mathrm{x}$ & $\mathrm{x}$ & $\mathrm{x}$ \\
\hline Serjania communis Cambess. & ten & - & - & - & $\mathrm{x}$ & - & - & - & $\mathrm{x}$ & $\mathrm{x}$ & - \\
\hline Serjania elegans Cambess. & ten & $\mathrm{x}$ & - & - & - & - & - & - & - & - & - \\
\hline Serjania fuscifolia Radlk. & ten & - & $\mathrm{x}$ & - & - & $\mathrm{x}$ & - & - & - & - & $\mathrm{x}$ \\
\hline Serjania glabrata Kunth. & ten & & $\mathrm{x}$ & - & - & - & - & - & - & - & - \\
\hline Serjania hebecarpa Benth. & ten & - & - & - & $\mathrm{x}$ & - & $\mathrm{x}$ & - & $\mathrm{x}$ & - & - \\
\hline Serjania laruotteana Cambess. & ten & - & $\mathrm{x}$ & - & $\mathrm{x}$ & $\mathrm{x}$ & $\mathrm{x}$ & $\mathrm{x}$ & - & - & $\mathrm{x}$ \\
\hline Serjania lethalis A.St.-Hil. & ten & - & $\mathrm{x}$ & $\mathrm{x}$ & $\mathrm{x}$ & $\mathrm{x}$ & $\mathrm{x}$ & - & - & - & $\mathrm{x}$ \\
\hline Serjania meridionalis Cambess. & ten & $\mathrm{x}$ & $\mathrm{x}$ & - & - & $\mathrm{x}$ & $\mathrm{x}$ & - & - & - & $\mathrm{x}$ \\
\hline Serjania multiflora Cambess. & ten & $\mathrm{x}$ & - & - & - & - & $\mathrm{x}$ & - & $\mathrm{x}$ & $\mathrm{x}$ & - \\
\hline Serjania paradoxa Radlk. & ten & - & - & - & - & - & - & - & - & - & $\mathrm{x}$ \\
\hline Serjania perulacea Radlk. & ten & - & - & - & - & - & - & - & - & $\mathrm{x}$ & - \\
\hline Serjania reticulata Cambess. & ten & $\mathrm{x}$ & $\mathrm{x}$ & - & - & - & - & - & $\mathrm{x}$ & - & - \\
\hline Serjania tristis Radlk. & ten & - & - & - & - & - & $\mathrm{x}$ & - & - & - & - \\
\hline Thinouia mucronata Radlk. & ten & - & - & - & - & - & - & $\mathrm{x}$ & $\mathrm{x}$ & - & - \\
\hline Urvillea laevis Radlk. & ten & - & $\mathrm{x}$ & - & $\mathrm{x}$ & $\mathrm{x}$ & $\mathrm{x}$ & $\mathrm{x}$ & $\mathrm{x}$ & $\mathrm{x}$ & $\mathrm{x}$ \\
\hline Urvillea ulmacea Kunth & ten & $\mathrm{x}$ & $\mathrm{x}$ & - & - & $\mathrm{x}$ & $\mathrm{x}$ & $\mathrm{x}$ & $\mathrm{x}$ & $\mathrm{x}$ & $\mathrm{x}$ \\
\hline Urvillea uniloba Radlk. & ten & - & - & - & $\mathrm{x}$ & $\mathrm{x}$ & - & - & - & - & - \\
\hline \multicolumn{12}{|l|}{ SMILACACEAE } \\
\hline Smilax brasiliensis Spreng. & ten & - & - & - & - & - & - & - & $\mathrm{x}$ & - & - \\
\hline Smilax campestris Griseb. & ten & $\mathrm{x}$ & - & - & - & $\mathrm{x}$ & $\mathrm{x}$ & - & $\mathrm{x}$ & - & - \\
\hline Smilax elastica Griseb. & ten & - & $\mathrm{x}$ & - & - & - & - & - & $\mathrm{x}$ & - & - \\
\hline Smilax fluminensis Steud. & ten & - & $\mathrm{x}$ & - & - & - & $\mathrm{x}$ & - & $\mathrm{x}$ & $\mathrm{x}$ & - \\
\hline Smilax polyantha Griseb. & ten & - & - & - & - & - & $\mathrm{x}$ & - & - & - & - \\
\hline Smilax rufescens Griseb. & ten & - & $\mathrm{x}$ & - & - & - & - & - & - & - & - \\
\hline Smilax spicata Vell. & ten & $\mathrm{x}$ & - & - & - & - & - & - & - & - & - \\
\hline SOLANACEAE & & & & & & & & & & & - \\
\hline Solanum alternatopinnatum Steud. & scr & - & $\mathrm{x}$ & - & $\mathrm{x}$ & - & - & - & - & - & - \\
\hline Solanum concinnum Sendtn. & scr & - & - & - & - & - & - & - & $\mathrm{x}$ & - & - \\
\hline Solanum pabstii L.B.Sm. \& Downs & scr & - & - & - & - & - & - & - & $\mathrm{x}$ & - & - \\
\hline Solanum swartzianum Roem. \& Schult. & scr & - & $\mathrm{x}$ & - & - & - & - & - & - & - & - \\
\hline Solanum wendlandii Hook.f. & scr & - & - & - & - & - & - & - & $\mathrm{x}$ & - & - \\
\hline \multicolumn{12}{|l|}{ TRIGONIACEAE } \\
\hline Trigonia nivea Cambess. & twi & $\mathrm{x}$ & - & - & - & $\mathrm{x}$ & - & - & $\mathrm{x}$ & $\mathrm{x}$ & - \\
\hline \multicolumn{12}{|l|}{ VERBENACEAE } \\
\hline Aloysia virgata (Ruiz \& Pav.) Pers. & scr & - & $\mathrm{x}$ & - & - & - & - & - & - & - & - \\
\hline Petrea volubilis L. & scr & $\mathrm{x}$ & $\mathrm{x}$ & - & - & $\mathrm{x}$ & $\mathrm{x}$ & - & $\mathrm{x}$ & $\mathrm{x}$ & - \\
\hline \multicolumn{12}{|l|}{ VIOLACEAE } \\
\hline Anchietea pyrifolia A.St.-Hil. & twi & $\mathrm{x}$ & - & $\mathrm{x}$ & - & - & - & - & - & $\mathrm{x}$ & - \\
\hline Anchietea salutaris A.St.-Hil. & twi & - & - & - & - & - & - & - & $\mathrm{x}$ & - & - \\
\hline \multicolumn{12}{|l|}{ VITACEAE } \\
\hline Cissus campestris (Baker) Planch. & ten & - & - & - & $\mathrm{x}$ & - & - & - & - & - & - \\
\hline Cissus erosa Rich. & ten & - & - & - & $\mathrm{x}$ & - & $\mathrm{x}$ & - & - & - & - \\
\hline Cissus serroniana (Glaz.) Lombardi & ten & - & $\mathrm{x}$ & - & - & - & - & - & - & - & - \\
\hline Cissus simsiana Schult. \& Schult.f. & ten & - & - & - & - & - & - & - & - & $\mathrm{x}$ & - \\
\hline Cissus subrhomboidea (Baker) Planch. & ten & - & - & - & - & - & $\mathrm{x}$ & - & - & - & - \\
\hline Cissus sulcicaulis (Baker) Planch. & ten & - & $\mathrm{x}$ & - & - & - & - & - & - & - & - \\
\hline Cissus verticillata (L.) Nicolson \& C.E.Jarvis & ten & - & $\mathrm{x}$ & - & - & $\mathrm{x}$ & $\mathrm{x}$ & - & $\mathrm{x}$ & - & - \\
\hline
\end{tabular}


Table 3. Characteristics of liana species composition in ten semideciduous forest sites of Southeastern Brazil. Sites legend as in Table 1 and Figure 1.

Tabela 3. Características da composição florística de lianas nos dez fragmentos de floresta estacional semidecidual estudados no sudeste do Brasil. Siglas dos fragmentos como na Figura 1 e Tabela 1.

\begin{tabular}{|c|c|c|c|c|c|c|c|c|c|c|c|}
\hline & \multicolumn{11}{|c|}{ Forest sites } \\
\hline & ANG & ARA & $\mathbf{B H}$ & PF & $\mathbf{R C}$ & $\mathbf{R P}$ & SC & SG & SV & VAS & Total \\
\hline \multicolumn{12}{|l|}{ Richness $^{\mathrm{a}}$} \\
\hline Liana species & 48 & 138 & 39 & 96 & 79 & 104 & 39 & 135 & 69 & 94 & 355 \\
\hline Families & 22 & 32 & 17 & 23 & 27 & 24 & 8 & 32 & 27 & 20 & 43 \\
\hline Exclusive species & 9 & 34 & 13 & 25 & 7 & 22 & 3 & 35 & 11 & 16 & 175 \\
\hline Tree species ${ }^{b}$ & 188 & 162 & - & 87 & 175 & - & 109 & 250 & 202 & - & - \\
\hline \multicolumn{12}{|l|}{ Richest families $^{\mathrm{a}, \mathrm{c}}$} \\
\hline Bignoniaceae & 9 & 29 & 9 & 15 & 16 & 13 & 18 & 22 & 12 & 24 & 45 \\
\hline Fabaceae & 2 & 3 & 3 & 21 & 6 & 11 & 5 & 7 & 6 & 3 & 42 \\
\hline Malpighiaceae & 3 & 10 & 10 & 8 & 3 & 10 & 4 & 17 & 5 & 13 & 36 \\
\hline Asteraceae & 6 & 17 & 3 & 1 & 5 & 3 & 0 & 12 & 7 & 7 & 31 \\
\hline Apocynaceae & 1 & 9 & 1 & 7 & 6 & 11 & 2 & 11 & 1 & 7 & 29 \\
\hline \multicolumn{12}{|l|}{ Climbing mechanisn ${ }^{\mathrm{a}}$} \\
\hline Tendril climbers & 21 & 57 & 13 & 39 & 38 & 45 & 25 & 48 & 32 & 43 & 121 \\
\hline Twiners & 18 & 56 & 19 & 49 & 32 & 53 & 9 & 70 & 28 & 41 & 178 \\
\hline Scramblers & 9 & 25 & 7 & 8 & 9 & 6 & 5 & 17 & 9 & 10 & 56 \\
\hline
\end{tabular}

${ }^{\mathrm{a}}=$ number of species ${ }^{\mathrm{b}}{ }^{=}$Number of tree species for referred site compiled from ANG: Torres (1989); ARA: Pagano \& Leitão Filho (1987); PF: Rezende et al. (2007); RC: Santos and Kinoshita (2003); SC: Hora (1999); SG: Morellato (1995); ${ }^{\mathrm{c}}=$ rankof the five richest family when considering all the sites pulled together, and the number of species of these families occurring in each forest site; - = non availiable information.

${ }^{\mathrm{a}}=$ número de espécies; ${ }^{\mathrm{b}}=$ número de espécies arbóreas encontradas no referido fragmento compilado de ANG: Torres (1989); ARA: Pagano \& Leitão Filho (1987); PF: Rezende et al. (2007); RC: Santos \& Kinoshita (2003); SC: Hora (1999); SG: Morellato (1995); ${ }^{\mathrm{c}}=$ ranking das famílias mais ricas quando considerado o total de espécies encontradas em todos os fragmentos e o número de espécies dessas famílias em cada uma das áreas; - = informação não disponível.

Cucurbitaceae (14), Passifloraceae (10), and Euphorbiaceae (8). These families contributed to $74.4 \%$ of the total species richness, whereas another 20 families were represented by only one or two species. The set of richest families varied little between the analysed forest sites (Table 3).

The contribution of the climbing species to the total number of wood species recorded on the examined forests ranged from $20.3 \%$ to $52.5 \%$ (Table 3 ). The exclusive species i.e., those occurring in only one forest site, accounted for $49.3 \%$ (175 species) of the total recorded (Table 3), $17.2 \%$ were present in two sites and $11 \%$ of the species occurred in three sites. Only one species, Macfadyena unguis-cati (L.) A.H.Gentry was common to all the studied forests.

The commonest climbing method within forest sites were main stem or branch twining, accounting for 178 species or $50.1 \%$ of the total recorded, followed by tendril climbing (121 species, $34.1 \%$ ), and scrambling (56 species, $15.8 \%$ ). Comparing the mean proportion of species $( \pm 95 \%$ confidence interval) in climbing mechanisms among forest sites, we found that $12.7 \%(+3.3-3.01 \%)$ were scramblers, $44.1 \%(+6.1-6.0 \%)$ were tendril climbers, and $42.7 \%(+6.5-6.4 \%)$ were twiners. The proportions of species in climbing mechanisms varied little among forest sites (Table 3). The highest proportion of twiners was observed at Santa Genebra (SG) and the lowest at São Carlos (SC) (Table 3).

The mean $( \pm$ SE) similarity among forest sites was $30.2 \%$ ( $+1.45-1.47 \%)$, ranging from 14.8 to $52.6 \%$. The NMS ordination of the ten forest sites based on presence/abcence of climbing species showed a cluster including 9 forest sites (Figure 2). Only Belo Horizonte $(\mathrm{BH})$ forest site appeared isolated from the others. The NMS ordination represented $81.4 \%$ of the variation in the dataset, with $59.2 \%$ loaded on axis 1 and $22.2 \%$ on axis 2 .

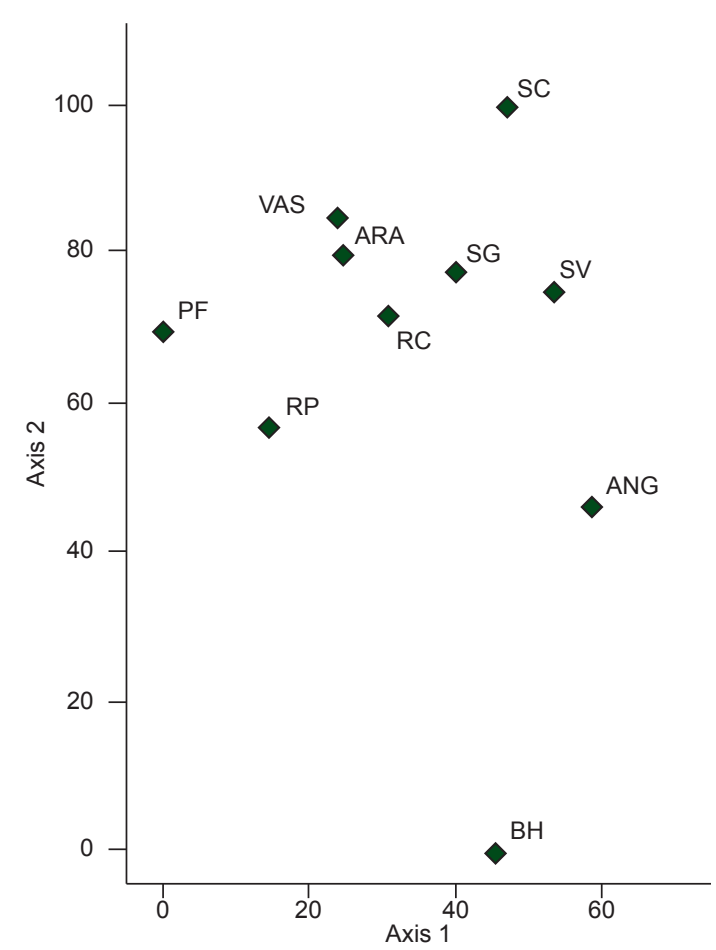

Figure 2. Two-dimensional MNS plot for the 10 semideciduous forest sites of southeastern Brazil. Sites legend as in Table 1 and Figure 1.

Figura 2. Ordenação bidimensional MNS obtida para os dez fragmentos de floresta estacional semidecidual estudados no sudeste do Brasil. Siglas dos fragmentos como na Figura 1 e Tabela 1. 


\section{Discussion}

Climbing species composition is described to be a function of stand age, geographical location, altitude and disturbance (DeWalt et al. 2000, Laurance et al. 2001, Parthasarathy et al. 2004). In our ordination analysis the geographical location appears to play a major role on the observed similarity among forests, since the only forests plotted separated from all others $(\mathrm{BH})$ is also the one located farthest apart geographically. Our results corroborate those found by Rezende \& Ranga 2005, which compared some of the floristic inventories (including $\mathrm{BH}$ ), using Jaccard index and clustering analysis UPGMA.

The list of the most speciose families on forest sites confirms partially Gentry's (1991) predictions that the communities of climbers in the neotropics may vary little from one forest site to another, in terms of floristic composition of families, with Bignoniaceae and/or Fabaceae almost always among the richest families. Bignoniaceae was the richest family in nine out of ten forest sites examined in the present study. Fabaceae was the second richest family when considering all the sites together, but it did not figured within the five richest families in every forest site, for instance in Araras (ARA) and Vassununga (VAS) this family presented only three species, figuring in tenth and eighth place in the ranking of the richest families, respectively. Gentry (1995) suggested that the Bignoniaceae tend to be the richest family in climbing species in neotropical forests with a strong dry season, such as the semideciduous forest of Southeastern Brazil. This family is the fifth richest family in climbing species in the neotropics (Gentry 1991). The largest Bignoniaceae tribe, Bignonieae, includes nearly half of the genera (47 of 104) and more than one third of the family's species (360 of 860) (Lohmann 2006). The entire tribe is composed exclusively or predominantly by tendrillate lianas and most of them are represented within Brazilian limits, having probably evolved there (Gentry 1980). It is possible that these factors are correlated with the high diversity of family Bignoniaceae on studied sites.

The proportion of species comprised by the ten richest families (74.4\%) is in accordance to what has been reported for the neotropics (64-69\%; Gentry 1991, DeWalt et al. 2000), and again confirms Gentry (1991) predictions.

Twining is frequently cited as the most common climbing method within liana communities, being the most species diverse and also the most abundant (Putz \& Chai 1987, Hegarty \& Caballé 1991, DeWalt et al. 2000, Laurance et al. 2001, Parthasarathy et al. 2004). Our results agreed with this when considering the whole pool of species, but when analyzing forest sites separately, we found that tendril species predominated over twiners in six locations. Campanello et al. (2007) also found tendril climbers as a majority of species in an Atlantic Forest patch in Argentina. The high proportion of tendril climbers in most of the studied sites is also an effect of the large amount of Bignoniaceae species in the data, since most of them are tendrilate species. Nonetheless, the supremacy of twinners and tendrilate species over scrambler species was found in all the studied sites and in most tropical forests elsewhere (Hegarty \& Caballé 1991). The absence of obviously specialized climbing organs in scrambler species is pointed out by Gentry (1991) as the possible reason for their relatively lower success than twinners and tendrilate species. According to Putz (1984), the main factor influencing the richness and abundance of different climbing mechanisms seems to be the support availability. Twiners can climb larger supports than do tendril climbers. Thus, forests with high density of small stems, characteristic of the earlier sucessional stages and gaps, tend to present a larger proportion of tendril climbers, while in mature forests twiners may be more successful due to their ability to climb larger supports (DeWalt et al. 2000, Laurance et al. 2001). Nonetheless, further investigation with appropriate designed experiments is required to address this question properly in semideciduous forests.

Climbing species contributed for up to $52.5 \%$ of the total wood plant species recorded on the studied sites. According to Gentry \& Dodson (1987) lianas can account for up to $25 \%$ of wood stem diversity on tropical forests. Thus, the climbers' contribution to the total wood species richness in some sites, such as RC, PF, and ARA, can be considered high. As stated by Udulutsch et al. (2004), it is evident that climbers may represent a great proportion of the species richness in semideciduous forests of Southeastern Brazil, which clearly enforces the need for further investigations and the conservation value of these forest fragments.

Comparisons among forest inventories differing in sampling methods are always difficult and should be interpreted with caution. Therefore, further investigation, especially with the inclusion of quantitative data, is strongly recommended to enhance the knowledge on lianas communities in semideciduous forests of Southeastern Brasil. Nevertheless, the high liana species contribution to the total wood plant richness found here, allied to the observation that almost half of species were present in just one site (49.3\%), and the low mean similarity between analyzed forests indicates the importance of liana communities to the plant diversity in semideciduous forests of Southeastern Brazil. Climbing species appeared to compose unique floras in each forest site, enhancing the regional diversity and the conservation value of forest remnants.

\section{Acknowledgements}

This research was carried out thanks to financial support of the Fundação de Amparo à Pesquisa do Estado de São Paulo and Conselho Nacional de Desenvolvimento Científico e Tecnológico (CNPq,). The authors want to express their appreciation for facilities and logistic supports provided by the Botanical Department of the State University of Campinas, and are pleased to acknowledge Professor Arne Anderberg from the Swedish Museum of Natural History for valuable comments on earlier versions of the manuscript. We are also indebted to two anonymous referees for their comments and suggestions that substantially improved the manuscript.

\section{References}

ANGIOSPERM PHYLOGENY GROUP - APG. 2003. An update of the Angiosperm Phylogeny Group classification for the orders and families of flowering plants: APG II. Botanical. Bot. J. Linnean Soc. 141(4):399436.

APPANAH, S. \& PUTZ, F.E. 1984. Climber abundance in virgin dipterocarp forest and the effect of pre-felling climber cutting on logging damage. Malaysian Forester 47(4):335-342.

BERNACCI, L.C. \& LEITÃO FILHO, H.F. 1996. Flora fanerogâmica da floresta da Fazenda São Vicente, Campinas, SP. Rev. Bras. Bot. 19(2):149-164.

CAMPANELLO, P.I., GARIBALDI, J.F., GENOVEVA, M.G. \& GOLDSTEIN, G. 2007. Lianas in a subtropical Atlantic Forest: host preference and tree growth. For. Ecol. Manage. 242(2):250-259.

DEWALT, S.J., SCHNITZER, S.A. \& DENSLOW, J.S. 2000. Density and diversity of lianas along a chronosequence in a central Panamanian lowland forest. J. Trop. Ecol. 16(1):1-9.

EMMONS, L.H. \& GENTRY, A.H. 1983. Tropical forest structure and the distribution of gliding and prehensil-tailed vertebrates. Am. Nat. 121(4):513-524.

GENTRY, A.H. 1980. BIGNONIACEAE. Part I (Crescentieae and Tourrettieae) In New York Botanical Garden (Flora Neotropica). New York, p.1-130. (Monograph 25). 
GENTRY, A.H. 1991. The distribution and evolution of climbing plants. In The biology of vines (F.E. Putz \& H.A. Mooney, eds), Cambridge University Press, Cambridge, p.3-49.

GENTRY, A.H. 1995. Diversity and floristic composition of neotropical dry forests. In Seasonally dry tropical forests (S.H. Bullock, H.A Mooney \& E. Medina, eds), Cambridge University Press, Cambridge, p.146-194.

GENTRY, A.H. \& DODSON, C. 1987. Contribution of nontrees to species richness of a tropical rain forest. Biotropica 19(2):149-156.

HEGARTY, E.E. 1991. Vine-host interactions. In The biology of vines (F.E. Putz \& H.A. Mooney, eds). Cambridge University Press, Cambridge, p.357-375.

HEGARTY, E.E. \& CABALLÉ, G. 1991. Distribuition and abundance of vines in forest communities. In The biology of vines (F.E. Putz \& H.A. Mooney, eds). Cambridge University Press, Cambridge, p.313-336.

HORA, R.C. 1999. Composição florística e aspectos da estrutura da comunidade de lianas em uma mata mesófila semidecídua na Fazenda Canchim, São Carlos - SP. Dissertação de Mestrado, Universidade Federal de São Carlos, São Carlos.

HORA, R.C. \& SOARES, J.J. 2002. Estrutura fitossociológica da comunidade de lianas em uma floresta estacional semidecidual na Fazenda Canchim, São Carlos, SP. Rev. Bras. Bot. 25(3):323-329.

LAURANCE, W.F. 1991. Edge effects in tropical forest fragments: application of a model for the design of nature reserves. Conserv. Biol. 57(2):205219.

LAURANCE, W.F. 1997. Hyper-disturbed parks: edge effects and the ecology of isolated rainforest reserves in tropical Australia. In Tropical forest remnants: ecology, management, and conservation of forest communities (W.F. Laurance \& R.O. Bierregaard, eds). University of Chicago Press, Chicago, p.71-83.

LAURANCE, W.F., PÉREZ-SALICRUP, D., DELAMÔNICA, P., FEARNSIDE, P.M., D'ANGELO, S., JEROLINSKI, A., POHL, L. \& LOVEJOY, T.E. 2001. Rain forest fragmentation and structure of amazonian liana communities. Ecology 82(1):105-116.

LOHMANN, L. 2006. Untangling the phylogeny of neotropical lianas (Bignonieae, Bignoniaceae). Amer. J. Bot. 93(2):304-319.

LOMBARDI, J.A., TEMPONI, L.G. \& LEITE, C.A. 1999. Mortality and diameter growth of lianas in a semideciduous forest fragment in Southeastern Brazil. Acta bot. Bras. 13(2):159-165.

MCCUNE, B. \& MEFFORD, M.J. 1999. PC-ORD. Multivariate Analysis of Ecological Data. MjM Software, Gleneden Beach, Oregon. (Version 4.34).

MORELLATO, L.P.C. \& LEITÃO FILHO, H.F. 1992. Padrões de frutificação e dispersão na serra do Japi. In História natural da Serra do Japi: ecologia e preservação de uma área florestal no sudeste do Brasil (L.P.C. Morellato, ed.). Editora da Unicamp/Fapesp, Campinas, p.112-141.

MORELLATO, L.P.C. \& LEITÃO FILHO, H.F. 1996. Reprodutive phenology of climbers in a Southeastern Brazilian Forest. Biotropica. 28(2):180191.

MORELLATO, L.P.C. 1995. Estações do ano na floresta. In Ecologia e preservação de uma floresta tropical urbana - Reserva de Santa Genebra (L.P.C. Morellato \& H.F. Leitão Filho, eds). Editora Unicamp. Campinas, p.37-41.

PAGANO, S.N. \& LEITÃO FILHO, H.F. 1987. Composição florística do estrato arbóreo da mata mesófila semidecídua no município de Rio Claro (Estado de São Paulo). Rev. Bras. Bot. 10(1):37-47.

PARTHASARATHY, N., MUTHURAMKUMAR, S. \& REDDY, M.S. 2004. Patterns of liana diversity in tropical evergreen forests of peninsular India. For. Ecol. Manage. 190(1):15-31.
PÉREZ-SALICRUP, D.R. \& BARKER, M.G. 2000. Effect of liana cutting on water potential and growth of Senna multijuga (Caesalpinioidae) trees in a Bolivian tropical forest. Oecologia 124(4):369-475.

PÉREZ-SALICRUP, D.R., SORK, V.L. \& PUTZ, F.E. 2001. Lianas and trees in a liana forest of amazonian Bolivia. Biotropica 33(1):34-47.

PHILLIPS, O.L., VASQUEZ, R., ARROYO, L., BAKER, T.R., KILLEEN, T., LEWIS, S., MALHI, Y., MONTEAGUDO, A., NEILL, D., NUNEZ VARGAS, P., ALEXIADES, M., CERON, C., DI FIORE, A., ERWIN, T., JARDIM, A., PALACIOS, W., SALDIAS, M. \& VINCETI, B. 2002. Increasing dominance of large lianas in Amazonian forests. Nature 418(6899): 770-774.

PHILLIPS, O.L., MARTINEZ, R.V., MENDOZA, A.M., BAKER, T.R. \& VARGAS, P.N. 2005 Large lianas as hyperdynamic elements of the tropical forest canopy. Ecology 85(5):1250- 1258.

PUTZ, F.E. 1984. The natural history of lianas on Barro Colorado Island, Panama. Ecology 68(6):1713-1724.

PUTZ, F.E. 1991. Silvicultural effects of lianas. In The biology of vines (F.E. Putz \& H.A. Mooney, eds). Cambridge University Press, Cambridge, p.493-501.

PUTZ, F.E. \& CHAI, P. 1987. Ecological studies of lianas in Lambir National Park, Sarawak, Malaysia. J. Ecol. 75(2):523-531.

REZENDE, A.A. \& RANGA, N.T. 2005. Lianas da Estação Ecológica do Noroeste Paulista, São José do Rio Preto/Mirassol, SP. Acta Bot. Bras. 19(2):273-279.

REZENDE, A.A., RANGA, N.T. \& PEREIRA, R.A.S. 2007. Lianas de uma floresta estacional semidecidual, Município de Paulo de Faria, Norte do Estado de São Paulo, Brasil. Rev. Bras. Bot. 30(3):451-461.

SANTOS, K. \& KINOSHITA, L.S. 2003. Flora arbustivo-arbórea do fragmento de floresta estacional semidecidual do Ribeirão Cachoeira, Município de Campinas - SP. Acta Bot. Bras. 17(3): 325-341.

SCHNITZER, S.A. \& CARSON, W.P. 2001. Treefall gaps and the maintenance of diversity in a tropical forest. Ecology 82(4):913-919.

SCHNITZER, S.A., DALLING, J.W. \& CARSON, W.P. 2000. The impact of lianas on tree regeneration in tropical forest canopy gaps: evidence for an alternative pathway of gap-phase regeneration. J. Ecol. 88(4):655-666.

STEVENS, G.C. 1987. Lianas as structural parasites: the Bursera simaruba example. Ecology 68(1):77-81.

STRANGHETTI, V. \& RANGA, N.T. 1998. Levantamento florístico das espécies vasculares de uma floresta estacional mesófila semidecídua da Estação Ecológica de Paulo de Faria, SP. Rev. Bras. Bot. 21(3):289298.

TIBIRIÇÁ, Y.J.A., COELHO, L.F.M. \& MOURA, L.C. 2006. Florística de lianas em um fragmento de floresta estacional semidecidual, Parque Estadual de Vassununga, Santa Rita do Passa Quatro, SP, Brasil. Acta Bot. Bras. 20(2):339-346.

TORRES, R.B. 1989. Estudos florísticos em mata secundária do sítio Ecológica de Angatuba; Angatuba (São Paulo). Dissertação de Mestrado, Universidade Estadual de Campinas, Campinas.

UDULUTSCH, R.G., ASSIS, M.A. \& PICCHI, D.G. 2004. Florística de trepadeiras numa floresta estacional semidecídua, Rio Claro - Araras, Estado de São Paulo, Brasil. Rev. Bras. Bot. 27(1):125-134.

VIDAL, E., JOHNS, J., GERWING, J., BARRETO, P. \& UHL, C. 1997. Vine management for reduced impact logging in eastern Amazonia. For. Ecol. Manage. 98(22):105-114. 